Article

\title{
Two Seas for One Great Diversity: Checklist of the Marine Heterobranchia (Mollusca; Gastropoda) from the Salento Peninsula (South-East Italy)
}

\author{
Giulia Furfaro ${ }^{1, *(\mathbb{D}}$, Fabio Vitale ${ }^{2,3}\left(\mathbb{D}\right.$, Cataldo Licchelli $^{2,4}$ and Paolo Mariottini ${ }^{5}$ \\ 1 Department of Biological and Environmental Sciences and Technologies-DiSTeBA, University of Salento, \\ I-73100 Lecce, Italy \\ 2 Salento Sommerso Association, I-73100 Lecce, Italy; fabio_vitale@tin.it (F.V.); \\ cataldo.licchelli@gmail.com (C.L.) \\ 3 Museum of Natural History of Salento, I-73021 Calimera-Lecce, Italy \\ 4 Cooperativa Hydra, I-73100 Lecce, Italy \\ 5 Department of Science, University of Roma Tre, I-00146 Rome, Italy; paolo.mariottini@uniroma3.it \\ * Correspondence: giulia.furfaro@unisalento.it
}

Received: 18 March 2020; Accepted: 24 April 2020; Published: 26 April 2020

\begin{abstract}
The Salento peninsula is a portion of the Italian mainland separating two distinct Mediterranean basins, the Ionian and the Adriatic seas. Several authors have studied the marine Heterobranchia (Mollusca, Gastropoda) fauna composition living in the Ionian Sea, but to date further knowledge regarding this interesting group of mollusks is still needed. Recent studies have corroborated the peculiarity of the Mediterranean Sea showing high levels of endemism and cryptic diversity. On the other hand, marine sea slugs have been revealed to be important indicators of the marine ecosystem's health, due to their species-specific diet that consist of a vast variety of sessile and benthic invertebrates. A baseline study of the marine Heterobranchia diversity is therefore a necessary step to reveal the hidden diversity and to monitor the possible presence of alien species. The present study shows results from approximately 600 scientific dives carried out during a nine-year period in all of the main submarine habitats of the studied area, while accounting for the marine Heterobranchia from both the Ionian and Adriatic Seas. With this contribution, the list of marine Heterobranchia inhabiting the Salento Peninsula rises to 160. Furthermore, it also reports, for the first time, the presence of one alien species and three new records for Italian waters. Ecological notes and geographical distribution for each added species are provided together with animal iconography, consisting mainly of in situ photographs, for species identification.
\end{abstract}

Keywords: diversity; sea slugs; nudibranchs; Mediterranean Sea; monitoring

\section{Introduction}

The Salento Peninsula (South-East Italy) in Puglia is a strip of land right on the border of the eastern Mediterranean Sea, made up of a large variety of habitats: rocky formations, open sandy beaches, marine caves, etc. The Salento Peninsula is a physically well-identified region, encompassing the innermost point of the Gulf of Taranto $\left(40^{\circ} 31^{\prime} 17.8^{\prime \prime} \mathrm{N} 17^{\circ} 06^{\prime} 10.7^{\prime \prime} \mathrm{E}\right)$ and the beach of Torre Santa Sabina di Ostuni $\left(40^{\circ} 45^{\prime} 52.3^{\prime \prime} \mathrm{N} 17^{\circ} 41^{\prime} 20.1^{\prime \prime} \mathrm{E}\right)$, with a length of 138 kilometers, calculated on the axis between Martina Franca $\left(40^{\circ} 42^{\prime} 17.7^{\prime \prime} \mathrm{N} 17^{\circ} 20^{\prime} 20.9^{\prime \prime} \mathrm{E}\right)$ and the Cape of Santa Maria di Leuca $\left(39^{\circ} 47^{\prime} 40.4^{\prime \prime} \mathrm{N} 18^{\circ} 22^{\prime} 05.0^{\prime \prime} \mathrm{E}\right)$. The maximum and minimum width are 54 and 33 kilometers, respectively, and the total coastal length is 365 kilometers [1-5]. This peninsula protrudes between two ecoregions of the Mediterranean Sea [6], the Northern Ionian, and the Southern Adriatic seas, which are conventionally separated by the dividing line passing through Otranto (according to the 
biogeographical zones described by Bianchi [7], i.e., zones 6 and 7, respectively). These two basins are characterised by peculiar and distinct main currents and submarine morphologies, resulting in very complex and dynamic ecosystems affected by seasonal fluctuations, which influences both the shallow and deep communities $[8,9]$. Therefore, this marine area could potentially host a high abundance of Heterobranchia species, due to their pelagic larval stage and diversified diet. Knowledge on diversity is a basic requisite to identify targets and to monitor species composition shifts over time, caused by natural or anthropogenic factors. In fact, the change in marine Heterobranchia fauna composition over time is considered a good ecological indicator of potential environmental modifications [10-14] and this peculiarity is particularly interesting in such a heterogeneous area of the Mediterranean. Furthermore, the capability of marine Heterobranchia to host biological compounds that are potentially interesting for biomedical applications, provides additional value to the study of this particular group of mollusks [14]. In fact, many species of marine Heterobranchia display aposematic vivacious warning colors to indicate that they contain defensive secondary metabolites that are sequestered, transformed from dietary sources, or synthesized de novo [15]. These compounds are used, mainly by nudibranchs, as a chemical defense from predation $[16,17]$ and have revealed to be an important source of diverse bioactive products used as effective analgesic, anti-inflammatory, antiviral, and anticancer drugs $[18,19]$. The Mediterranean marine diversity is therefore revealed to be interesting, mainly due to the presence of cryptic or endemic species. In fact, several species once considered to be widespread across the Atlantic and the Mediterranean were shown to be a complex of cryptic species, many of which are endemic to the Mediterranean (e.g., [20-23]). This trend is also true for marine Heterobranchia, as demonstrated by recent molecular papers that helped to unravel such cryptic diversity [24-29]. In this context, studies focused on the production of species lists from different geographical areas are needed as an essential starting point to unveil this hidden diversity. In the past decade, the key works on marine Heterobranchia in the area under investigation have been published by Perrone [30-37], who mainly provided data regarding the species inhabiting the Ionian side of the Salento peninsula (Gulf of Taranto), and only provided ecological notes on a small number of species. Recently, Onorato and Belmonte [38] reviewed the biodiversity assessment of the marine submerged caves in the Salento peninsula, which includes some heterobranchs, while Micaroni et al. [39] published a check-list of Heterobranchia from the Ionian locality of Tricase, adding 20 species to Perrone's previous lists [30-37]. Finally, several published papers that did not have proper checklists, added new records of single species sampled from the Salento Peninsula [29,40-44], contributing to the increase of lists of species known from this Apulian area. However, to date, there is no published checklist of marine Heterobranchia fauna from the Adriatic side of the Salento peninsula. During the last ten years, there has been an increasing interest in marine observation by underwater photographers $[45,46]$. This has allowed the possibility to extend the research on fauna, to detect lesser known species, and to create a collaboration network between scientists and amateurs who are experts in this field. The so-called Citizen Science dedicated to the Heterobranchia is an important support in expanding the body of knowledge on this group of gastropods, by providing field observations on bathymetric distribution, seasonality [47], egg deposition, reproductive behavior and trophic niche, and by making the data immediately available to the scientific community through web-based social networks [48]. In the last decade, due to the combined efforts of professional underwater photographers and acknowledged systematic experts in sea slugs, many new records and ecological observations on the Salento Peninsula marine Heterobranchia have been collected. Taking all of these points into account, the present study had the following aims to: (i) contribute to the Salento Peninsula marine Heterobranchia checklist with new records from the sublittoral waters, considering both sides of this Peninsula, the Ionian and the Adriatic sides, for the first time; (ii) provide ecological notes and local distribution for each new recorded species; (iii) show in situ photographs of the live animals to document species identification. 


\section{Materials and Methods}

The geographical area under investigation was a stretch of more than $270 \mathrm{~km}$ of coastline around the Salento Peninsula in Southern Italy (Figure 1). With 600 scuba dives in the past 9 years, different benthic habitats were surveyed, some of which were included in the European legislative context (EU WFD, EU Habitat Directive, EU MSFD): pre-coralligenous and coralligenous assemblages, soft-bottom substrates, Posidonia oceanica (Linnaeus) Delile, 1813 meadows, and algal biocoenosis on rocky substrates. All sampled sites were georeferenced (Table 1) to provide accurate data of the studied locations for future monitoring and comparison. When possible, ecological observation and in situ photographs of the individuals recorded were performed and catalogued for species identification. The Scuba dives ( $0-40 \mathrm{~m}$ depth) took place all year round, almost every week, during daylight and at night, between 2011 and 2019. Specimens between 2 and $5 \mathrm{~mm}$ in length were photographed alive in the laboratory, in Petri capsules illuminated by a series of low voltage LED lamps, with $6500 \mathrm{~K}$ bulbs. The camera used for filming was a tripod mounted Nikon D7100, with $60 \mathrm{~mm}$ micro Nikkor or $105 \mathrm{~mm}$ micro Nikkor optics, a series of extension rings and additional lenses. The underwater photographic or videography equipment (F.V.) was a Nikon D7000 body, $60 \mathrm{~mm}$ micro Nikkor, or $105 \mathrm{~mm}$ micro Nikkor optics, extension rings inside an Isotta housing, as well as additional wet lenses SubSee +10 diopter, two underwater flashes Inon z240, and two LED lamps of great luminous power. Or alternatively, (C.L.) a Canon 600D body in a Nauticam 600D housing, equipped with the following lenses-for macro photography and in some cases for micro subjects, the Canon $60 \mathrm{~mm}$ USM Macro, and Canon 100 mm USM Macro were used, in addition to wet lenses SubSee +5 and +10 diopter; for wide angle photography, the Tokina $10-17 \mathrm{~mm}$ fisheye lens was used. The light source was provided by a couple of Inon strobes z240 and a single focus light I-Torch Video Pro 3. The systematics and the validity of names were checked with the help of the Word Register of Marine Species [49]. Species identification was obtained by morphological investigation and a subsequent consultation of the existent literature $([29,41,44]$ and other references cited in the present work), guide books $[50,51]$ and websites [48,52,53]. In the case of Berthellina cf. edwardsii, the shell from an individual (Voucher RM3_1865) was extracted and used to confirm the identification as this anatomical feature is commonly considered to be diagnostic for this species. The shell was removed and dissolved in a $10 \% \mathrm{NaOH}$ solution, then rinsed in water, dried, and mounted for examination by optical microscopy, following the same protocol described by Furfaro et al. [54]. Voucher numbers were assigned to the collected individuals that were selected for future molecular analyses; samples were preserved in $95 \%$ alcohol and stored in the Department of Science at the Roma Tre University (Rome, Italy) (Table 2). Finally, a comparison between species as recorded by previous authors (Perrone [30-37] (A), Onorato and Belmonte [38] (B), Micaroni et al. [39] (C)) and the present study (D) was carried out and reported in Table 2 with new records highlighted in bold letters. An ethical approach in this research was also carried out by complying with the restrictions in term of collected sample size, environmental survey of the collection sites, use of hand-net picking of specimens (harmless and not destructive) as well as complying with local, regional, national, and international rules, and regulations for access to biodiversity, sustainable use, and benefit sharing (Convention on Biological Diversity and its Nagoya Protocol, national regulations). 


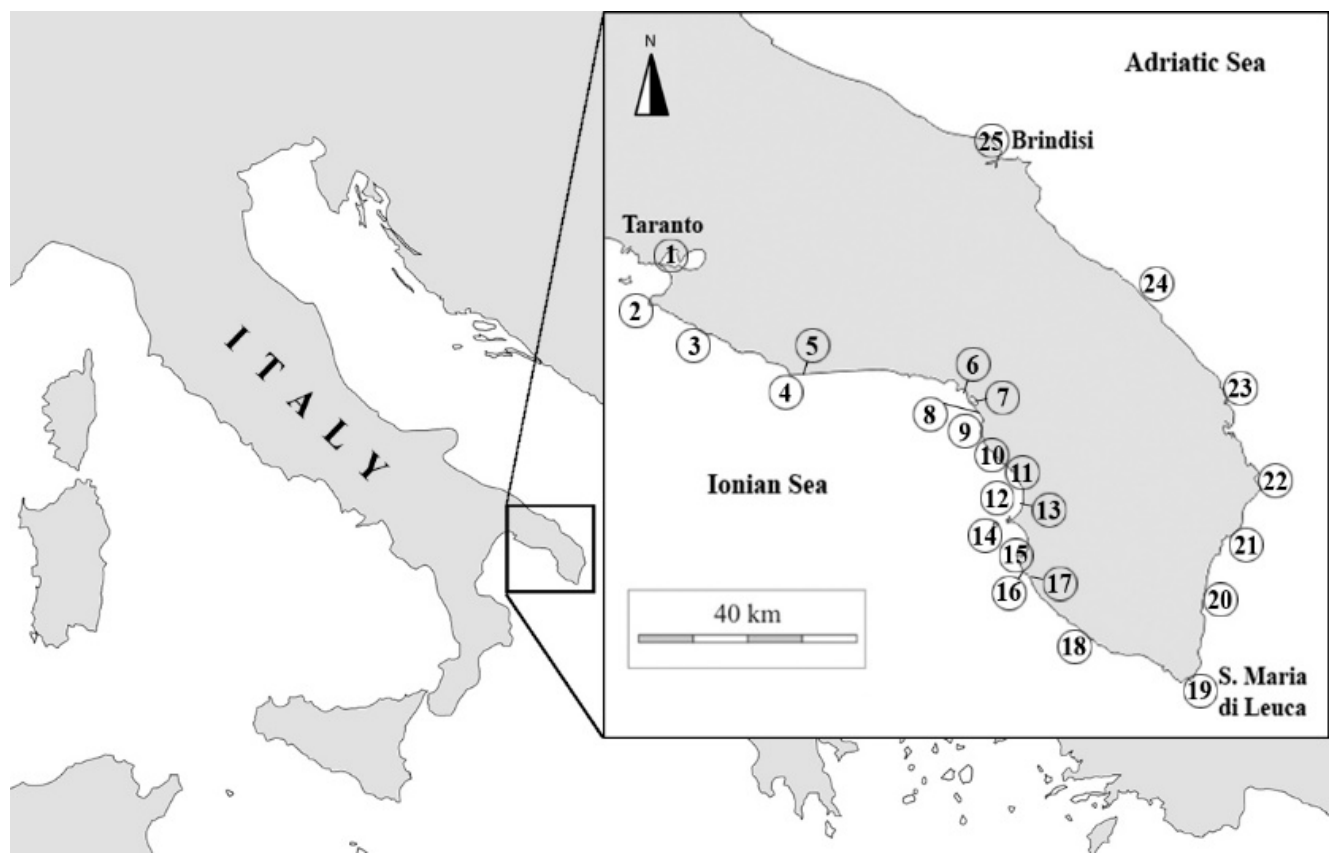

Figure 1. Map of the Salento Peninsula indicating the sampling localities; the box highlights the Salento Peninsula at a higher magnification. Numbers refer to sampling stations reported in Table 1.

Table 1. Number, name, geographic coordinates (latitude and longitude), and the depth range of the sampled stations.

\begin{tabular}{|c|c|c|c|c|}
\hline $\mathbf{N}^{\circ}$ & Station & Latitude & Longitude & Depth \\
\hline 1 & Mar Piccolo, Taranto (Ionian Sea) & $40^{\circ} 28^{\prime} 53.62^{\prime \prime} \mathrm{N}$ & $17^{\circ} 16^{\prime} 00.91^{\prime \prime} \mathrm{E}$ & $1-10 \mathrm{~m}$ \\
\hline 2 & Capo S. Vito, Taranto (Ionian Sea) & $40^{\circ} 24^{\prime} 35.85^{\prime \prime} \mathrm{N}$ & $17^{\circ} 12^{\prime} 06.07^{\prime \prime} \mathrm{E}$ & $1-20 \mathrm{~m}$ \\
\hline 3 & Porto Pirrone, Taranto (Ionian Sea) & $40^{\circ} 21^{\prime} 27.79^{\prime \prime} \mathrm{N}$ & $17^{\circ} 19^{\prime} 40.93^{\prime \prime} \mathrm{E}$ & $4-20 \mathrm{~m}$ \\
\hline 4 & Torre Ovo, Taranto (Ionian Sea) & $40^{\circ} 17^{\prime} 29.18^{\prime \prime} \mathrm{N}$ & $17^{\circ} 30^{\prime} 11.20^{\prime \prime} \mathrm{E}$ & $1-20 \mathrm{~m}$ \\
\hline 5 & Campomarino, Taranto (Ionian Sea) & $40^{\circ} 17^{\prime} 34.76^{\prime \prime} \mathrm{N}$ & $17^{\circ} 31^{\prime} 41.48^{\prime \prime} \mathrm{E}$ & $1-20 \mathrm{~m}$ \\
\hline 6 & Porto Cesareo I. Conigli, Lecce (Ionian Sea) & $40^{\circ} 15^{\prime} 32.15^{\prime \prime} \mathrm{N}$ & $17^{\circ} 52^{\prime} 57.59^{\prime \prime} \mathrm{E}$ & $1-15 \mathrm{~m}$ \\
\hline 7 & Porto Cesareo, Lecce (Ionian Sea) & $40^{\circ} 14^{\prime} 51.48^{\prime \prime} \mathrm{N}$ & $17^{\circ} 54^{\prime} 33.51^{\prime \prime} \mathrm{E}$ & $0-1 \mathrm{~m}$ \\
\hline 8 & S. Isidoro, Lecce (Ionian Sea) & $40^{\circ} 13^{\prime} 15.51^{\prime \prime} \mathrm{N}$ & $17^{\circ} 55^{\prime} 29.27^{\prime \prime} \mathrm{E}$ & $1-5 \mathrm{~m}$ \\
\hline 9 & Torre Inserraglio, Lecce (Ionian Sea) & $40^{\circ} 10^{\prime} 41.44^{\prime \prime} \mathrm{N}$ & $17^{\circ} 55^{\prime} 51.53^{\prime \prime} \mathrm{E}$ & $1-20 \mathrm{~m}$ \\
\hline 10 & Santa Caterina di Nardò, Lecce (Ionian Sea) & $40^{\circ} 08^{\prime} 05.87^{\prime \prime} \mathrm{N}$ & $17^{\circ} 59^{\prime} 15.53^{\prime \prime} \mathrm{E}$ & $1-15 \mathrm{~m}$ \\
\hline 11 & Santa Maria al Bagno, Lecce (Ionian Sea) & $40^{\circ} 07^{\prime} 30.58^{\prime \prime} \mathrm{N}$ & $17^{\circ} 59^{\prime} 46.43^{\prime \prime} \mathrm{E}$ & $0-6 \mathrm{~m}$ \\
\hline 12 & O.R. Gallipoli, Lecce (Ionian Sea) & $40^{\circ} 06^{\prime} 04.68^{\prime \prime} \mathrm{N}$ & $17^{\circ} 58^{\prime} 04.22^{\prime \prime} \mathrm{E}$ & $36 \mathrm{~m}$ \\
\hline 13 & Gallipoli, Lecce (Ionian Sea) & $40^{\circ} 04^{\prime} 34.70^{\prime \prime} \mathrm{N}$ & $17^{\circ} 59^{\prime} 50.25^{\prime \prime} \mathrm{E}$ & $1-20 \mathrm{~m}$ \\
\hline 14 & Gallipoli - Isola S. Andrea, Lecce (Ionian Sea) & $40^{\circ} 02^{\prime} 36.77^{\prime \prime} \mathrm{N}$ & $17^{\circ} 57^{\prime} 01.64^{\prime \prime} \mathrm{E}$ & $5-20 \mathrm{~m}$ \\
\hline 15 & Gallipoli Pizzo, Lecce (Ionian Sea) & $39^{\circ} 59^{\prime} 57.64^{\prime \prime} \mathrm{N}$ & $17^{\circ} 59^{\prime} 27.36^{\prime \prime} \mathrm{E}$ & $1-15 \mathrm{~m}$ \\
\hline 16 & Marina Mancaversa, Lecce (Ionian Sea) & $39^{\circ} 58^{\prime} 58.04^{\prime \prime} \mathrm{N}$ & $18^{\circ} 00^{\prime} 18.58^{\prime \prime} \mathrm{E}$ & $1-5 \mathrm{~m}$ \\
\hline 17 & Torre Suda, Lecce (Ionian Sea) & $39^{\circ} 56^{\prime} 27.95^{\prime \prime} \mathrm{N}$ & $18^{\circ} 02^{\prime} 17.36^{\prime \prime} \mathrm{E}$ & $1-5 \mathrm{~m}$ \\
\hline 18 & Ugento, Lecce (Ionian Sea) & $39^{\circ} 52^{\prime} 36.70^{\prime \prime} \mathrm{N}$ & $18^{\circ} 05^{\prime} 25.14^{\prime \prime} \mathrm{E}$ & $5-30 \mathrm{~m}$ \\
\hline 19 & Santa Maria di Leuca, Lecce (Ionian Sea) & $39^{\circ} 48^{\prime} 18.38^{\prime \prime} \mathrm{N}$ & $18^{\circ} 22^{\prime} 42.56^{\prime \prime} \mathrm{E}$ & $1-20 \mathrm{~m}$ \\
\hline 20 & Tricase, Lecce (Adriatic Sea) & $39^{\circ} 55^{\prime} 51.31^{\prime \prime} \mathrm{N}$ & $18^{\circ} 23^{\prime} 47.92^{\prime \prime} \mathrm{E}$ & $1-30 \mathrm{~m}$ \\
\hline 21 & Porto Miggiano, Lecce (Adriatic Sea) & $40^{\circ} 01^{\prime} 46.72^{\prime \prime} \mathrm{N}$ & $18^{\circ} 27^{\prime} 01.30^{\prime \prime} \mathrm{E}$ & $1-25 \mathrm{~m}$ \\
\hline 22 & Otranto, Lecce (Adriatic Sea) & $40^{\circ} 08^{\prime} 18.52^{\prime \prime} \mathrm{N}$ & $18^{\circ} 30^{\prime} 47.65^{\prime \prime} \mathrm{E}$ & $1-40 \mathrm{~m}$ \\
\hline 23 & Roca, Lecce (Adriatic Sea) & $40^{\circ} 17^{\prime} 50.18^{\prime \prime} \mathrm{N}$ & $18^{\circ} 24^{\prime} 49.69^{\prime \prime} \mathrm{E}$ & $1-6 \mathrm{~m}$ \\
\hline 24 & Frigole, Lecce (Adriatic Sea) & $40^{\circ} 26^{\prime} 20.52^{\prime \prime} \mathrm{N}$ & $18^{\circ} 14^{\prime} 46.56^{\prime \prime} \mathrm{E}$ & $0-1 \mathrm{~m}$ \\
\hline 25 & Brindisi (Adriatic Sea) & $40^{\circ} 39^{\prime} 54.11^{\prime \prime} \mathrm{N}$ & $17^{\circ} 57^{\prime} 34.81^{\prime \prime} \mathrm{E}$ & $1-8 \mathrm{~m}$ \\
\hline
\end{tabular}


Table 2. List of species of Heterobranchia occurring around the Salento peninsula; localities are numbered according to Figure 1, with new records in column D, highlighted in bold. Column $\mathbf{A}=$ Perrone [30-37]; B = Onorato and Belmonte [38]; C = Micaroni et al. [39]; and D = Present study.

\begin{tabular}{|c|c|c|c|c|c|}
\hline Taxonomy & $\mathbf{A}$ & B & $\mathrm{C}$ & D & Vouchers \\
\hline \multicolumn{6}{|c|}{ Pleurobranchida } \\
\hline \multicolumn{6}{|l|}{ Family Pleurobranchidae Gray, 1827} \\
\hline Pleurobranchus membranaceus (Montagu, 1803) & 16 & & & & \\
\hline Pleurobranchus testudinarius Cantraine, 1835 & & & & 1,17 & \\
\hline Berthellina cf. edwardsii & & & & $1,4,6,10,13,15$ & RM3_1865 \\
\hline Berthella aurantiaca (Risso, 1818) & $8,9,19,23$ & & & & \\
\hline Berthella elongata (Cantraine, 1836) & 16 & & & & \\
\hline Berthella ocellata (delle Chiaje, 1830) & 8 & & & 5,6 & \\
\hline Berthella plumula (Montagu, 1803) & 16 & & & & \\
\hline Berthella stellata (Risso, 1826) & 9 & & & 22 & \\
\hline Berthellina citrina (Rüppell \& Leuckart, 1828) & 18 & & & & \\
\hline \multicolumn{6}{|l|}{ Family Pleurobranchaeidae Pilsbry, 1896} \\
\hline Pleurobranchaea meckeli (Blainville, 1825) & 16 & & 11 & 5,6 & \\
\hline \multicolumn{6}{|c|}{ Nudibranchia - Doridina } \\
\hline \multicolumn{6}{|l|}{ Family Calycidorididae Roginskaya, 1972} \\
\hline Diaphorodoris luteocincta (M. Sars, 1870) & & & & 6 & \\
\hline Diaphorodoris papillata Portmann \& Sandmeier, 1960 & & & 11 & $5,6,10$ & \\
\hline \multicolumn{6}{|l|}{ Family Onchidorididae Gray, 1927} \\
\hline Adalaria proxima (Alder \& Hancock, 1854) & 21 & & & & \\
\hline Knoutsodonta albonigra (Pruvot-Fol, 1951) & 20,24 & & & & \\
\hline \multicolumn{6}{|l|}{ Family Goniodorididae H. \& A. Adams, 1854} \\
\hline Goniodoris castanea Alder \& Hancock, 1845 & 20 & & & 1 & \\
\hline $\begin{array}{l}\text { Okenia longiductis Pola M, Paz-Sedano S, Macali A, } \\
\text { Minchin D, Marchini A, Vitale F, } 2019 \text { [41] }\end{array}$ & & & & 1,4 & \\
\hline Okenia mediterranea (von Ihering, 1886) [41] & & & & 6 & \\
\hline $\begin{array}{l}\text { Okenia problematica Pola M, Paz-Sedano S, Macali A, } \\
\text { Minchin D, Marchini A, Vitale F, } 2019 \text { [41] }\end{array}$ & & & & 7 & \\
\hline Trapania lineata Haefelfinger, 1960 & & & & $4-6,10,13,15$ & $\begin{array}{l}\text { RM3_1042, } \\
\text { RM3_1048, } \\
\text { RM3_1077 }\end{array}$ \\
\hline Trapania maculata Haefelfinger, 1960 & & & & $4-6,10,13,15$ & RM3_1076 \\
\hline \multicolumn{6}{|l|}{ Family Polyceridae Alder \& Hancock, 1845} \\
\hline Crimora papillata Alder \& Hancock, 1862 & & & & $5,6,11$ & \\
\hline Kaloplocamus ramosus (Cantraine, 1835) & & & 11 & & \\
\hline Polycera elegans (Bergh, 1894) & & & & 1 & \\
\hline Polycera hedgpethi Marcus, 1964 & & & & 1 & \\
\hline Polycera quadrilineata (O. F. Müller, 1776) & 3,16 & & 11 & $1,3-6,10,13,15$ & RM3_1065 \\
\hline Thecacera pennigera (Montagu, 1815) & & & & 1 & \\
\hline \multicolumn{6}{|l|}{ Family Aegiridae P. Fischer, 1883} \\
\hline Aegires punctilucens (d'Orbigny, 1837) & 4 & & & & \\
\hline \multicolumn{6}{|l|}{ Family Cadlinidae Bergh, 1891} \\
\hline Aldisa banyulensis Pruvot-Fol, 1951 & 23,24 & & & 6 & \\
\hline \multicolumn{6}{|l|}{ Family Chromodorididae Bergh, 1891} \\
\hline Felimare fontandraui (Pruvot-Fol, 1951) & & & & 6,8 & $\begin{array}{l}\text { RM3_1039, } \\
\text { RM3_1040, } \\
\text { RM3_1099, } \\
\text { RM3_1100 }\end{array}$ \\
\hline Felimare orsinii (Vérany, 1846) & & & & $8,12,13,15$ & \\
\hline Felimare picta (Philippi, 1836) & 19 & 13 & 11 & $\begin{array}{c}1,4-6 \\
8,10,12,13,15\end{array}$ & $\begin{array}{l}\text { RM3_1041, } \\
\text { RM3_1052, } \\
\text { RM3_1053 }\end{array}$ \\
\hline Felimare tricolor (Cantraine, 1835) & 5 & & 11 & $\begin{array}{c}1,4-6 \\
8,10,12,13,15\end{array}$ & $\begin{array}{l}\text { RM3_1074, } \\
\text { RM3_1075 }\end{array}$ \\
\hline Felimare villafranca (Risso, 1818) & 16 & & 11 & $1,5,6$ & $\begin{array}{l}\text { RM3_1231, } \\
\text { RM3_1232 }\end{array}$ \\
\hline
\end{tabular}


Table 2. Cont.

\begin{tabular}{|c|c|c|c|c|c|}
\hline Taxonomy & $\mathbf{A}$ & B & $\mathrm{C}$ & $\mathrm{D}$ & Vouchers \\
\hline Felimida binza (Ev. Marcus \& Er. Marcus, 1963) & & & & 15 & \\
\hline Felimida krohni (Vérany, 1846) & & 13 & 11 & $4-6,13$ & $\begin{array}{l}\text { RM3_1061, } \\
\text { RM3_1068 }\end{array}$ \\
\hline Felimida luteorosea (Rapp, 1827) & 16 & & 11 & 1,6 & \\
\hline Felimida purpurea (Risso, 1831) & 4 & & & 6 & \\
\hline \multicolumn{6}{|l|}{ Family Dorididae Rafinesque, 1815} \\
\hline Doris ocelligera (Bergh, 1881) & & & 11 & 3 & \\
\hline Doris pseudoargus Rapp, 1827 & 19 & & & & \\
\hline Doris verrucosa Linnaeus, 1758 & 19 & & & 1 & \\
\hline \multicolumn{6}{|l|}{ Family Discodorididae Bergh, 1891} \\
\hline Atagema rugosa Pruvot-Fol, 1951 & 19 & & & & \\
\hline Baptodoris cinnabarina Bergh, 1884 & 8,20 & & & 22,6 & \\
\hline Discodoris stellifera (Vayssière, 1903) & 9,19 & & & 15 & \\
\hline Gargamella perezi (Llera \& Ortea, 1982) & 19,24 & & & & \\
\hline Geitodoris bonosi Ortea \& Ballesteros, 1981 & 3 & & & & \\
\hline Geitodoris portmanni (Schmekel, 1972) & 20 & & & & \\
\hline Jorunna tomentosa (Cuvier, 1804) & $3,10,20$ & & & 1,13 & \\
\hline Paradoris indecora (Bergh, 1881) & 3,24 & & & & \\
\hline Peltodoris atromaculata Bergh, 1880 & 8 & & 11 & ALL & $\begin{array}{l}\text { RM3_1054, } \\
\text { RM3_1056, } \\
\text { RM3_1057 }\end{array}$ \\
\hline Peltodoris sordii Perrone, 1989 & 3 & & & & \\
\hline Platydoris argo (Linnaeus, 1767) & 8 & & 11 & $5,6,10,13$ & \\
\hline Rostanga anthelia Perrone, 1991 & 4 & & & & \\
\hline Rostanga rubra (Risso, 1818) & 20,23 & & & & \\
\hline Taringa armata Swennen, 1961 & 22 & & & & \\
\hline Taringa pinoi Perrone, 1985 & 20,24 & & & & \\
\hline Tayuva lilacina (Gould, 1852) & 3 & & & 1,22 & \\
\hline \multicolumn{6}{|l|}{ Family Phyllidiidae Rafinesque, 1814} \\
\hline Phyllidia flava Aradas, 1847 & 19 & & 11 & $4-6,9,10,13$ & $\begin{array}{l}\text { RM3_1049, } \\
\text { RM3_1055, } \\
\text { RM3_1058 }\end{array}$ \\
\hline \multicolumn{6}{|l|}{ Family Dendrodorididae O'Donoghue, 1924} \\
\hline Dendrodoris grandiflora (Rapp, 1827) & 16 & & 11 & $1,3,5,6$ & \\
\hline Dendrodoris limbata (Cuvier, 1804) & $\begin{array}{l}1,16 \\
19\end{array}$ & & & $1,3,5,6$ & \\
\hline Doriopsilla areolata Bergh, 1880 & 17,23 & & & $3,4,6$ & \\
\hline \multicolumn{6}{|c|}{ Nudibranchia - Cladobranchia } \\
\hline \multicolumn{6}{|l|}{ Family Tritoniidae Lamarck, 1809} \\
\hline Marionia blainvillea (Risso, 1818) & 9 & & & 19 & \\
\hline Tritonia manicata Deshayes, 1853 & 8,16 & & 11 & 5,6 & \\
\hline Tritonia nilsodhneri Marcus Ev., 1983 & & & & 9 & \\
\hline Tritonia striata Haefelfinger, 1963 & 16 & & & 5,6 & \\
\hline \multicolumn{6}{|l|}{ Family Hancockiidae MacFarland, 1923} \\
\hline Hancockia uncinata (Hesse, 1872) & 16 & & & & \\
\hline \multicolumn{6}{|l|}{ Family Scyllaeidae Alder \& Hancock, 1855} \\
\hline Scyllaea pelagica Linnaeus, 1758 & 19 & & & & \\
\hline \multicolumn{6}{|l|}{ Family Tethydidae Rafinesque, 1815} \\
\hline Melibe viridis Kelaart, 1858 [55] & & & & $1,5,6$ & \\
\hline Tethys fimbria Linnaeus, 1767 & 16 & & & 6,13 & \\
\hline \multicolumn{6}{|l|}{ Family Dotidae Gray, 1853} \\
\hline Doto acuta Schmekel \& Kress, 1977 & & & 11 & & \\
\hline Doto cervicenigra Ortea \& Bouchet, 1989 & & & & 3,6 & \\
\hline Doto floridicola Simroth, 1888 & & & & 13 & \\
\hline Doto fragaria Ortea \& Bouchet, 1989 [56] & & & & 6 & \\
\hline Doto koenneckeri Lemche, 1976 & & & 11 & 13 & \\
\hline Doto paulinae Trinchese, 1881 & & & 11 & 6 & \\
\hline Doto pygmaea Bergh, 1871 & & & 11 & & \\
\hline
\end{tabular}


Table 2. Cont.

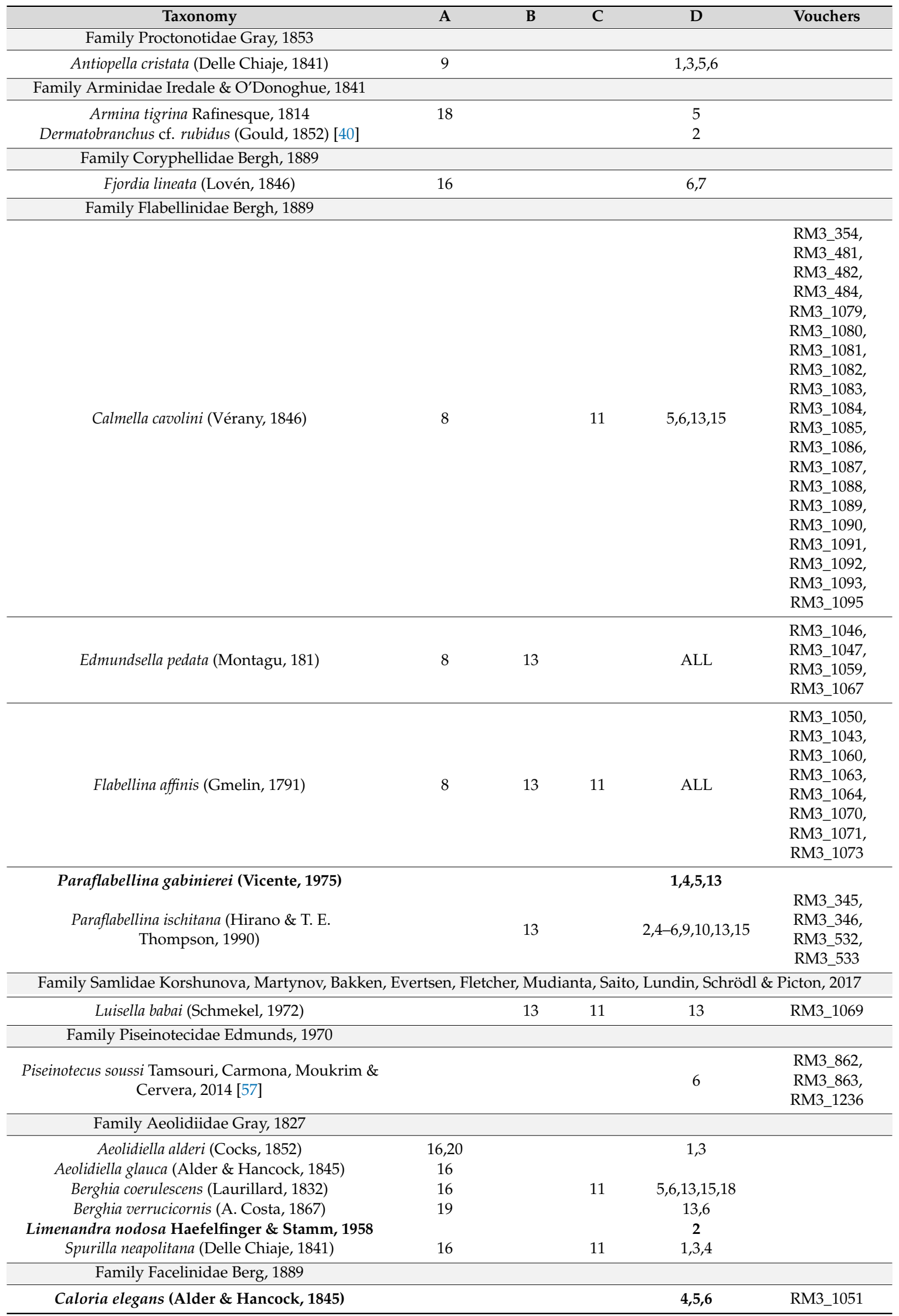


Table 2. Cont.

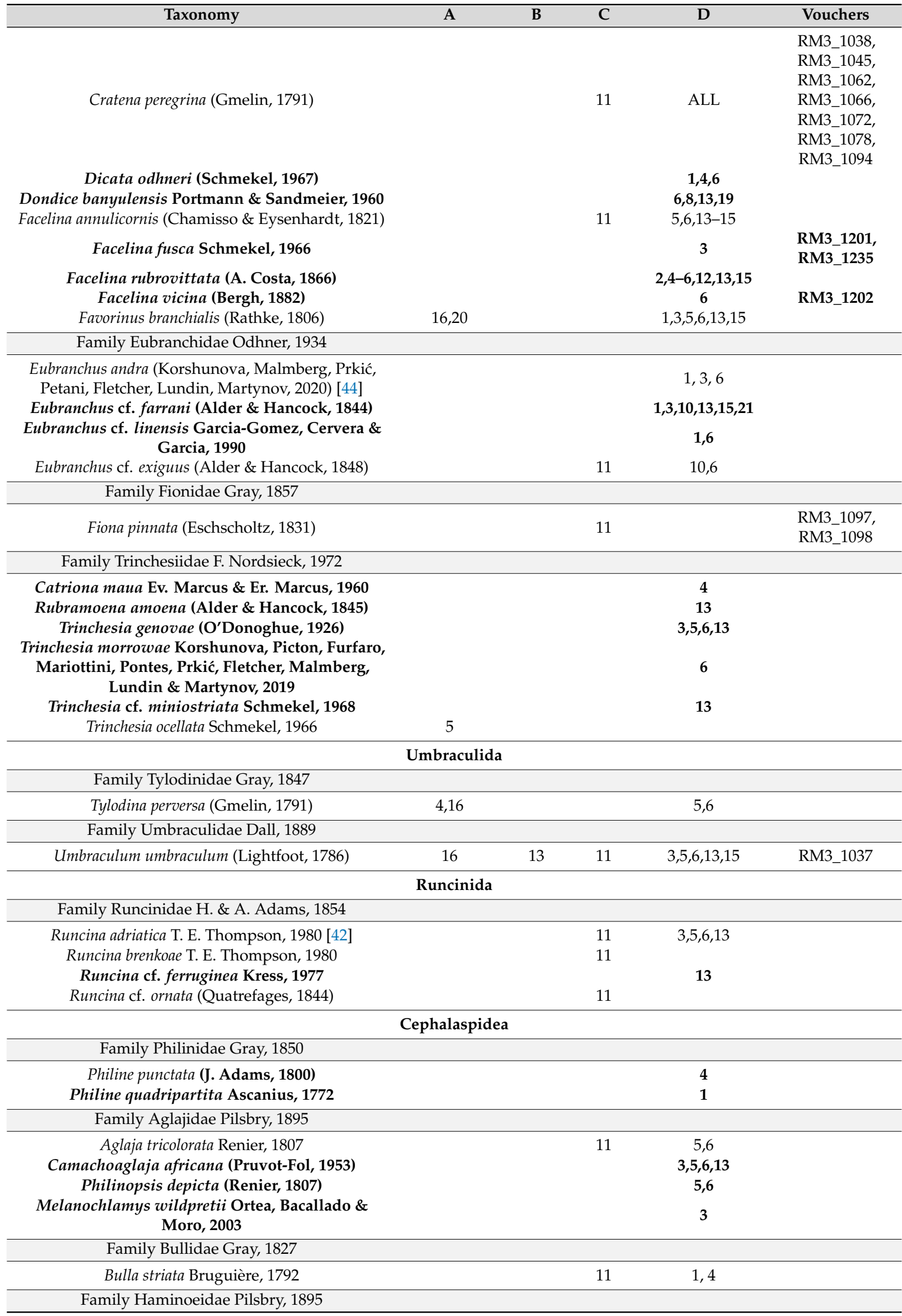


Table 2. Cont.

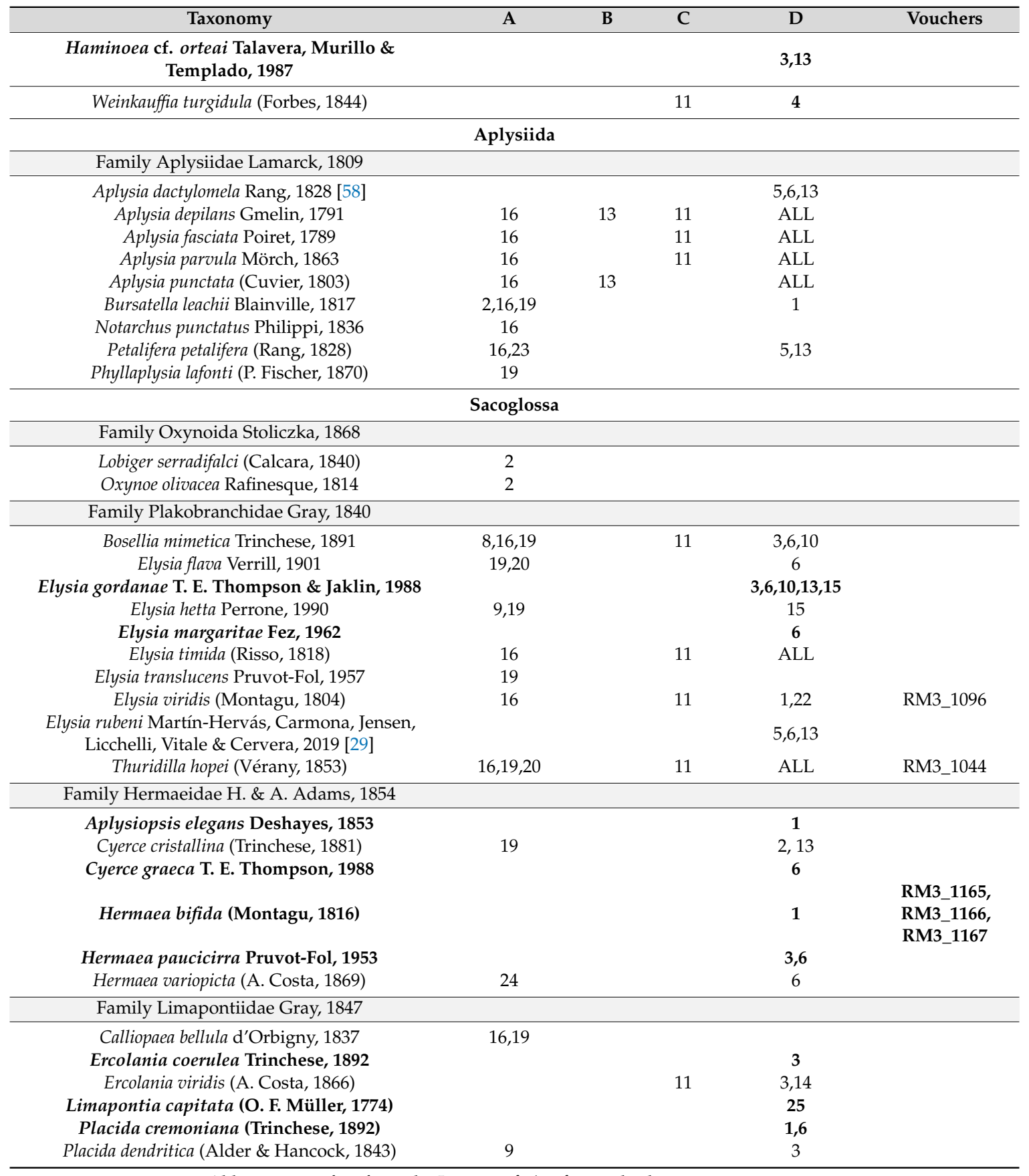

Abbreviation cf. is from the Latin confer/conferatur, both meaning compare.

\section{Results}

The present checklist reports 160 marine Heterobranchia species from the Salento Peninsula (Table 2) consisting of: 10 Pleurobranchida, 9 Cephalaspidea, 4 Runcinida, 2 Umbraculida, 9 Aplisiida, 24 Sacoglossa, and 102 Nudibranchia (50 Doridina, 52 Cladobranchia). This contribution added 45 species (Tables 2 and 3) to the marine heterobranchs fauna known from this area, so far, and in particular, 2 species belonging to Pleurobranchida, 6 Cephalaspidea, 1 Runcinida, 9 Sacoglossa, and 27 Nudibranchia (9 Doridina, 18 Cladobranchia). One alien species, Polycera hedgpethi Er. (Marcus, 1964), was reported for the first time in the studied area. This work reported for the first time the presence of Elysia margaritae (Fez, 1962), Haminoea cf. orteai (Talavera, Murillo and Templado, 1987), and Rubramoena amoena (Alder \& Hancock, 1845), in Italian waters. Table 3 provides the species list with 
ecological remarks of all added species. Furthermore, an extensive photographic catalogue mainly consisting of pictures taken in situ is provided in Figures 2-9.

Table 3. List of the newly recorded Heterobranchia from the Salento peninsula; species names, relative figure numbers, ecological notes, and phenotypical variability for each added species are reported. Abundance is indicated as number of specimens.

\begin{tabular}{|c|c|c|c|c|c|}
\hline & Species Name & Figure & Ecological Notes & $\begin{array}{c}\text { Phenotypical } \\
\text { Variability }\end{array}$ & Abundance \\
\hline 1 & $\begin{array}{l}\text { Pleurobranchus } \\
\text { testudinarius } \\
\text { Cantraine, } 1835\end{array}$ & Figure 2A & $\begin{array}{l}\text { On soft bottom during diurnal dive. } \\
\text { Depth: } 25 \mathrm{~m}\end{array}$ & - & $1-2$ \\
\hline 2 & $\begin{array}{c}\text { Berthellina cf. } \\
\text { edwardsii (Vayssière, } \\
\text { 1897) }\end{array}$ & Figure 2B,C & $\begin{array}{l}\text { Usually living singularly or in groups } \\
\text { under stones, in small shaded } \\
\text { crevices or in dark caves. } \\
\text { Depth: } 0-15 \mathrm{~m}\end{array}$ & $\begin{array}{l}\text { Body color ranging } \\
\text { from light } \\
\text { creamy-yellow to } \\
\text { reddish-orange }\end{array}$ & $>100$ \\
\hline 3 & $\begin{array}{l}\text { Diaphorodoris } \\
\text { luteocincta } \\
\text { (M. Sars, 1870) }\end{array}$ & Figure 2D & $\begin{array}{c}\text { This sedentary species usually lives } \\
\text { near bryozoan colonies (cf. Nolella } \\
\text { stipata Gosse, } 1855 \text { ). } \\
\text { Depth: } 0-3 \mathrm{~m}\end{array}$ & $\begin{array}{l}\text { The specimens of this } \\
\text { species show the red } \\
\text { spot on the dorsum } \\
\text { which varies in shape } \\
\text { and size }\end{array}$ & $3-10$ \\
\hline 5 & $\begin{array}{l}\text { Trapania maculata } \\
\text { Haefelfinger, } 1960\end{array}$ & Figure $2 \mathrm{~F}$ & $\begin{array}{c}\text { Often sympatric with the congeneric } \\
\text { T. lineata. } \\
\text { Depth: } 0-30 \mathrm{~m}\end{array}$ & - & $>100$ \\
\hline 6 & $\begin{array}{l}\text { Crimora papillata } \\
\text { Alder \& Hancock, } \\
1862\end{array}$ & Figure 2G,H & $\begin{array}{l}\text { Found all year long, in shady } \\
\text { pre-coralligenous shallow waters or } \\
\text { coastal caves. Often in association } \\
\text { with encrusting bryozoans on which } \\
\text { their egg masses are laid. } \\
\text { Depth: } 0-10 \mathrm{~m}\end{array}$ & $\begin{array}{l}\text { Color of the notum } \\
\text { variable from light } \\
\text { yellow to ochre }\end{array}$ & $>100$ \\
\hline 7 & $\begin{array}{l}\text { Polycera elegans } \\
\text { (Bergh, 1894) }\end{array}$ & Figure 3A,B & $\begin{array}{l}\text { Observed during the winter months, } \\
\text { in shallow waters. } \\
\text { Depth: } 5 \mathrm{~m}\end{array}$ & $\begin{array}{l}\text { Very typical body color } \\
\text { pattern, showing blue } \\
\text { spots differing in size } \\
\text { and number between } \\
\quad \text { individuals }\end{array}$ & $11-30$ \\
\hline 9 & $\begin{array}{l}\text { Felimare fontandraui } \\
\text { (Pruvot-Fol, 1951) }\end{array}$ & Figure 3E,F & $\begin{array}{c}\text { Observed in large assemblages at the } \\
\text { end of spring, associated with the } \\
\text { sponge Dysidea avara (Schmidt, 1862). } \\
\text { Depth: } 7 \mathrm{~m}\end{array}$ & $\begin{array}{l}\text { Even if this species } \\
\text { shows a variable } \\
\text { phenotype [26], the } \\
\text { Salentine specimens } \\
\text { have constant body } \\
\text { color pattern }\end{array}$ & $31-100$ \\
\hline 10 & $\begin{array}{l}\text { Felimare orsinii } \\
\text { (Vérany, 1846) }\end{array}$ & Figure 3G & $\begin{array}{l}\text { In large groups mating and feeding } \\
\text { on black sponges. Found between } \\
\text { April and July. } \\
\text { Depth: } 0-15 \mathrm{~m}\end{array}$ & - & $31-100$ \\
\hline 11 & $\begin{array}{l}\text { Felimida binza } \\
\text { (Ev. Marcus \& Er. } \\
\text { Marcus, 1963) }\end{array}$ & Figure $3 \mathrm{H}$ & $\begin{array}{l}\text { On a rocky substrates. Found during } \\
\text { September. } \\
\text { Depth: } 5-7 \mathrm{~m}\end{array}$ & - & $1-2$ \\
\hline 12 & $\begin{array}{l}\text { Tritonia nilsodhneri } \\
\text { Marcus Ev., } 1983\end{array}$ & Figure $4 \mathrm{~A}$ & $\begin{array}{l}\text { Found on the yellow gorgonian } \\
\text { Eunicella cavolinii Koch, } 1887 . \\
\text { Depth: } 30 \mathrm{~m}\end{array}$ & $\begin{array}{l}\text { Very mimetic } \\
\text { morphotype that can } \\
\text { consistently vary from } \\
\text { dark brown to pale } \\
\text { yellow or white }\end{array}$ & $1-2$ \\
\hline 13 & $\begin{array}{l}\text { Doto cervicenigra } \\
\text { Ortea \& Bouchet, } \\
1989\end{array}$ & Figure 4B & $\begin{array}{l}\text { This small species (few millimetres) is } \\
\text { found from winter to early spring on } \\
\text { hydrozoans colonies of Aglaophenia } \\
\text { Lamouroux, } 1812 \text {. } \\
\text { Depth: } 0-3 \mathrm{~m}\end{array}$ & - & $11-30$ \\
\hline
\end{tabular}


Table 3. Cont.

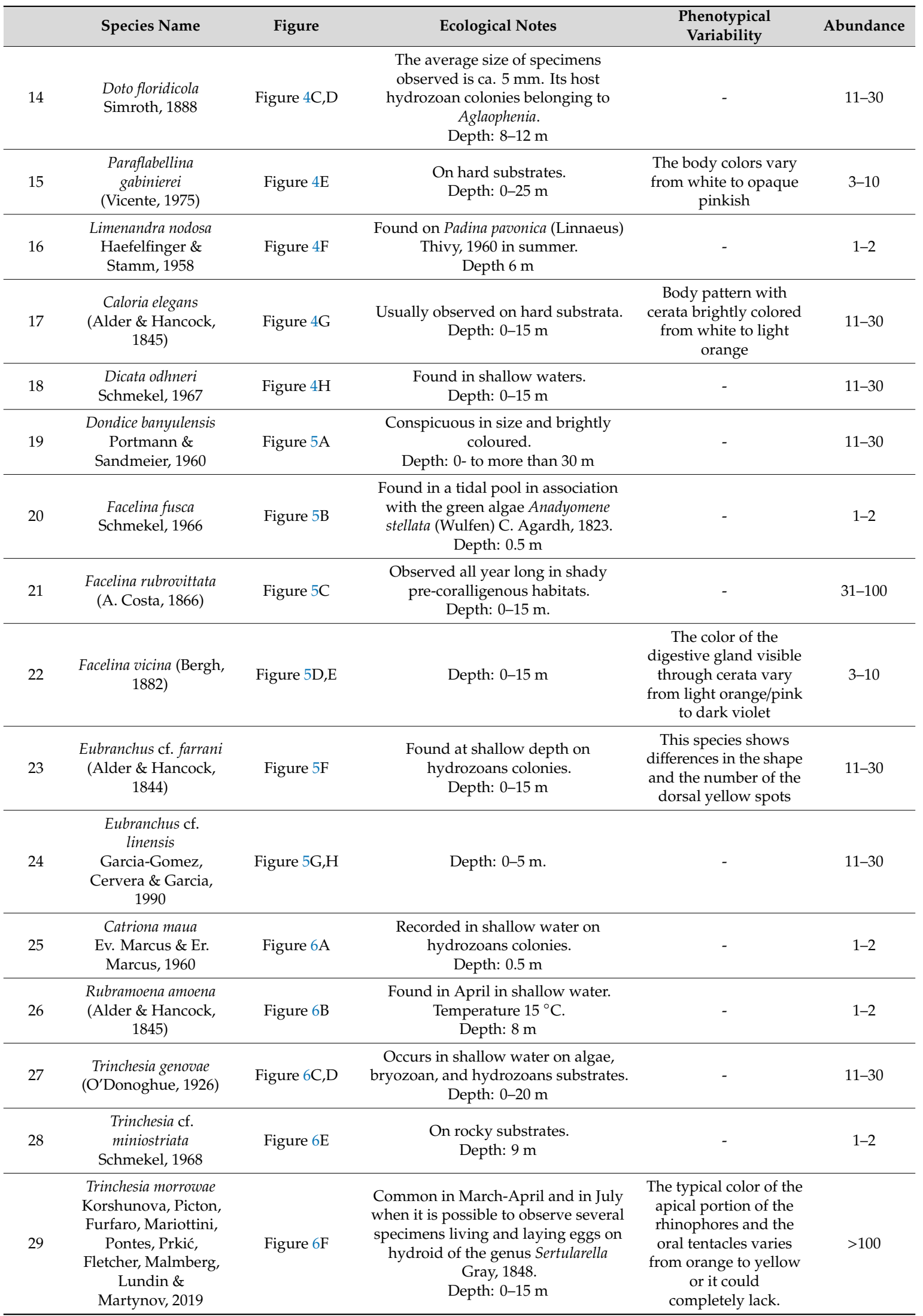


Table 3. Cont

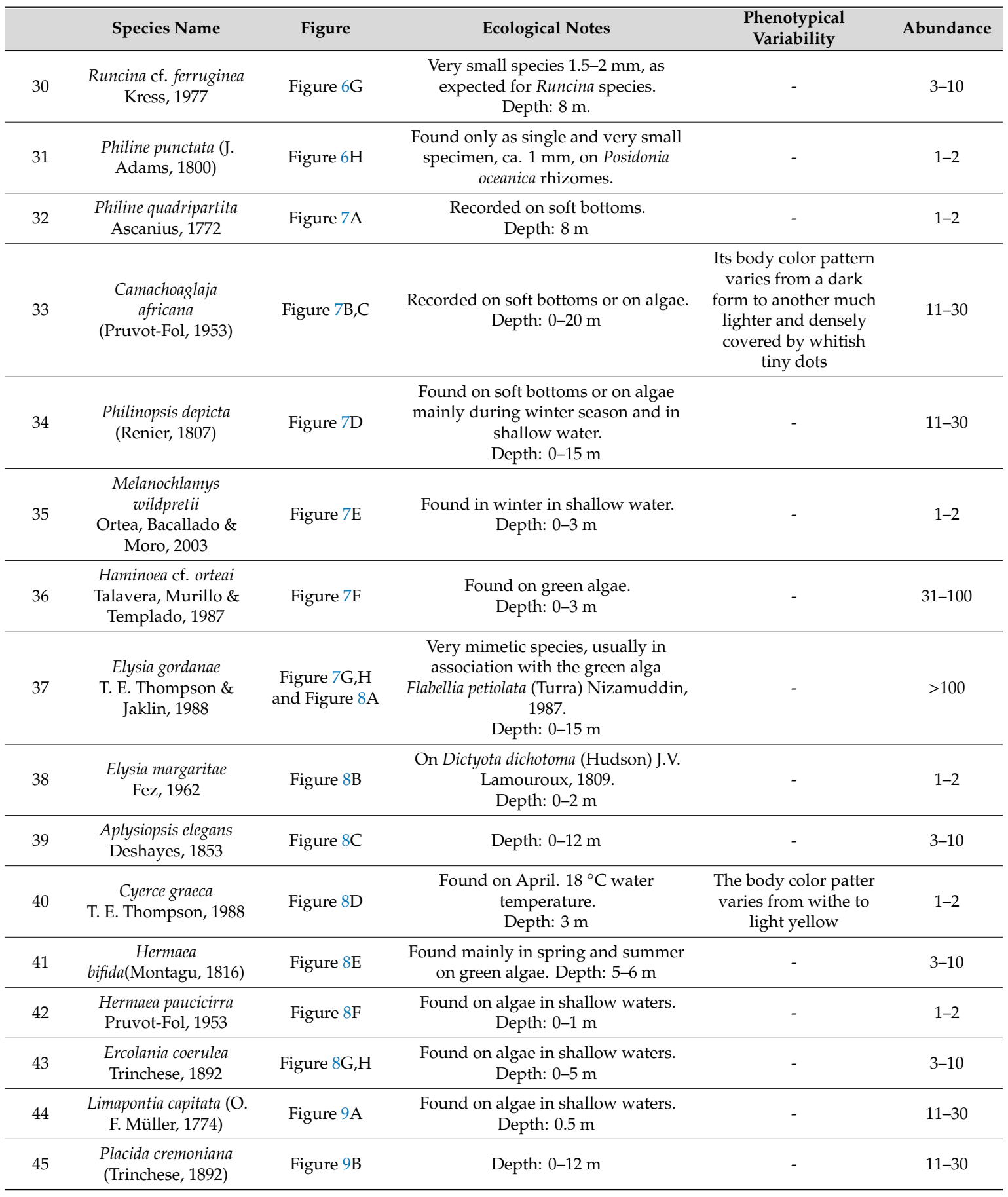



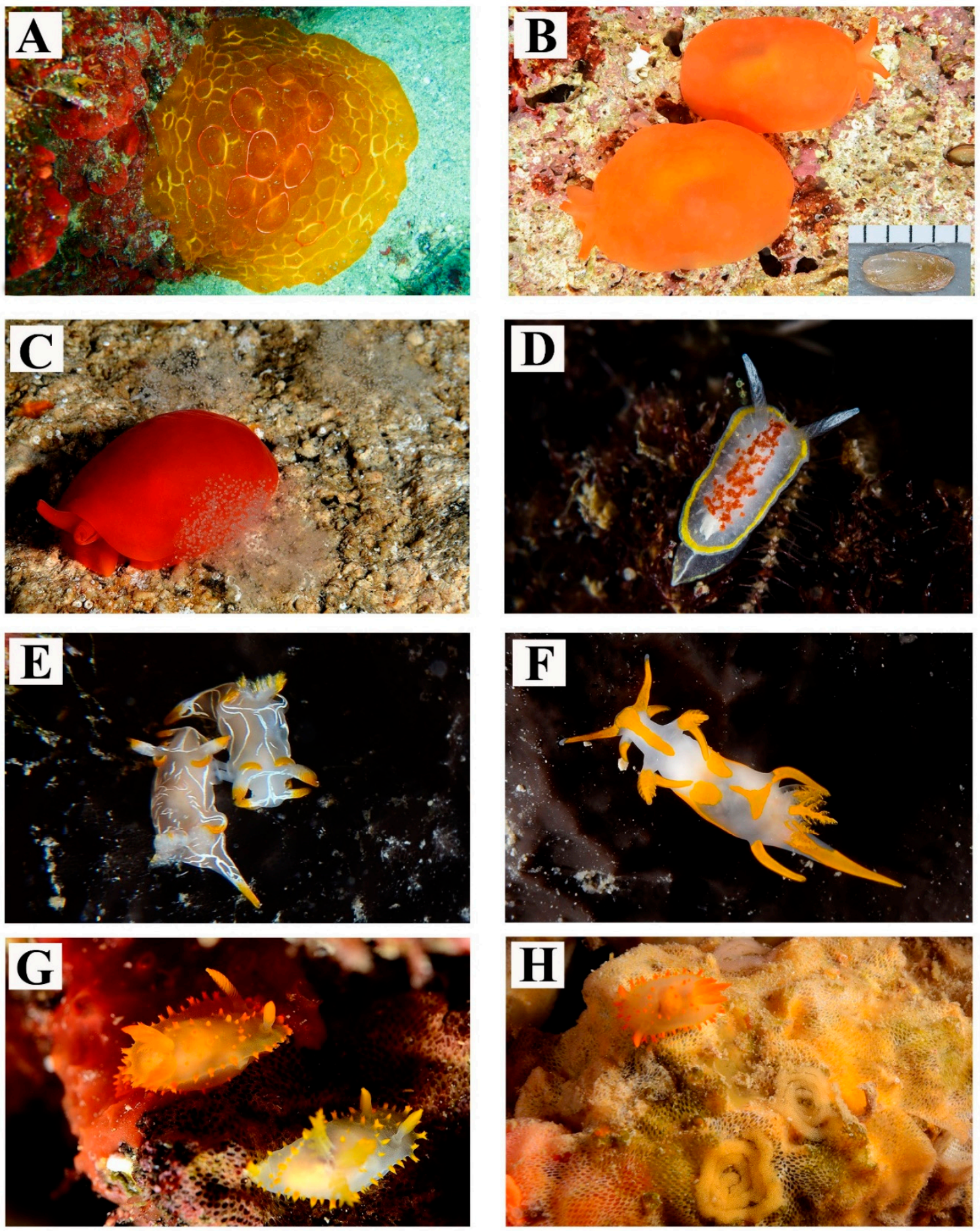

Figure 2. (A) Pleurobranchus testudinarius. St. 3 (B) Berthellina cf. edwardsii. St. 11. In the right-low corner, the internal shell (length: $4.2 \mathrm{~mm}$ ). (C) Berthellina cf. edwardsii. St. 11 (D) Diaphorodoris luteocincta. St. 11 (E) Trapania lineata, mating individuals. St. 11 (F) Trapania maculata. St. 11 (G) Crimora papillata. St. $11(\mathbf{H})$ Crimora papillata, with spawn. St. 11. 

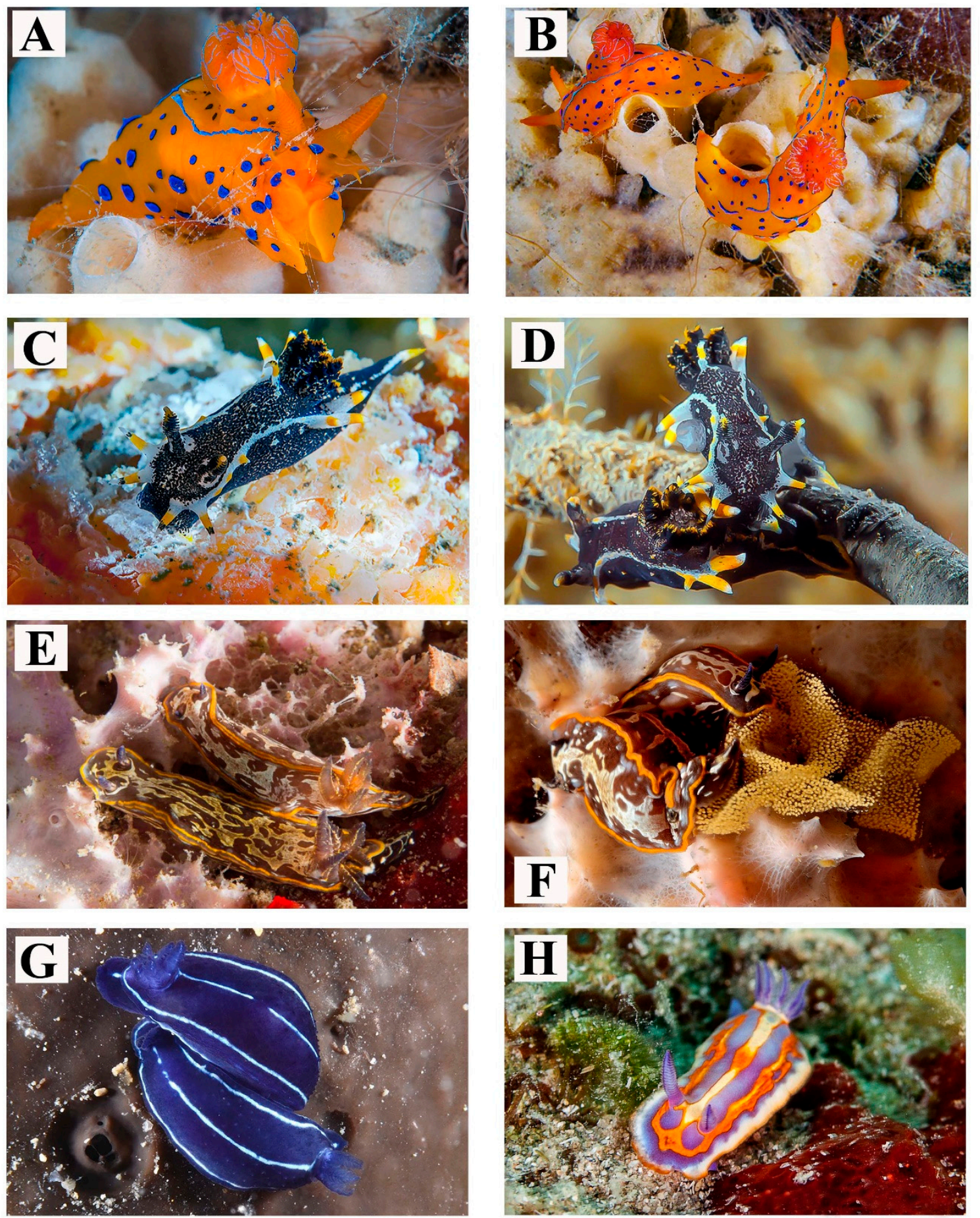

Figure 3. (A) Polycera elegans. St. 1 (B) Polycera elegans. St. 1 (C) Polycera hedgpethi. St. 1 (D) Polycera hedgpethi. Two individuals after mating. The reproductive openings are clearly visible in the specimen on the upper portion. St. 1 (E) Felimare fontandraui. St. 11 (F) Felimare fontandraui, mating individuals with spawn. St. 11 (G) Felimare orsinii, mating individuals. St. 14 (H) Felimida binza. St. 25. 

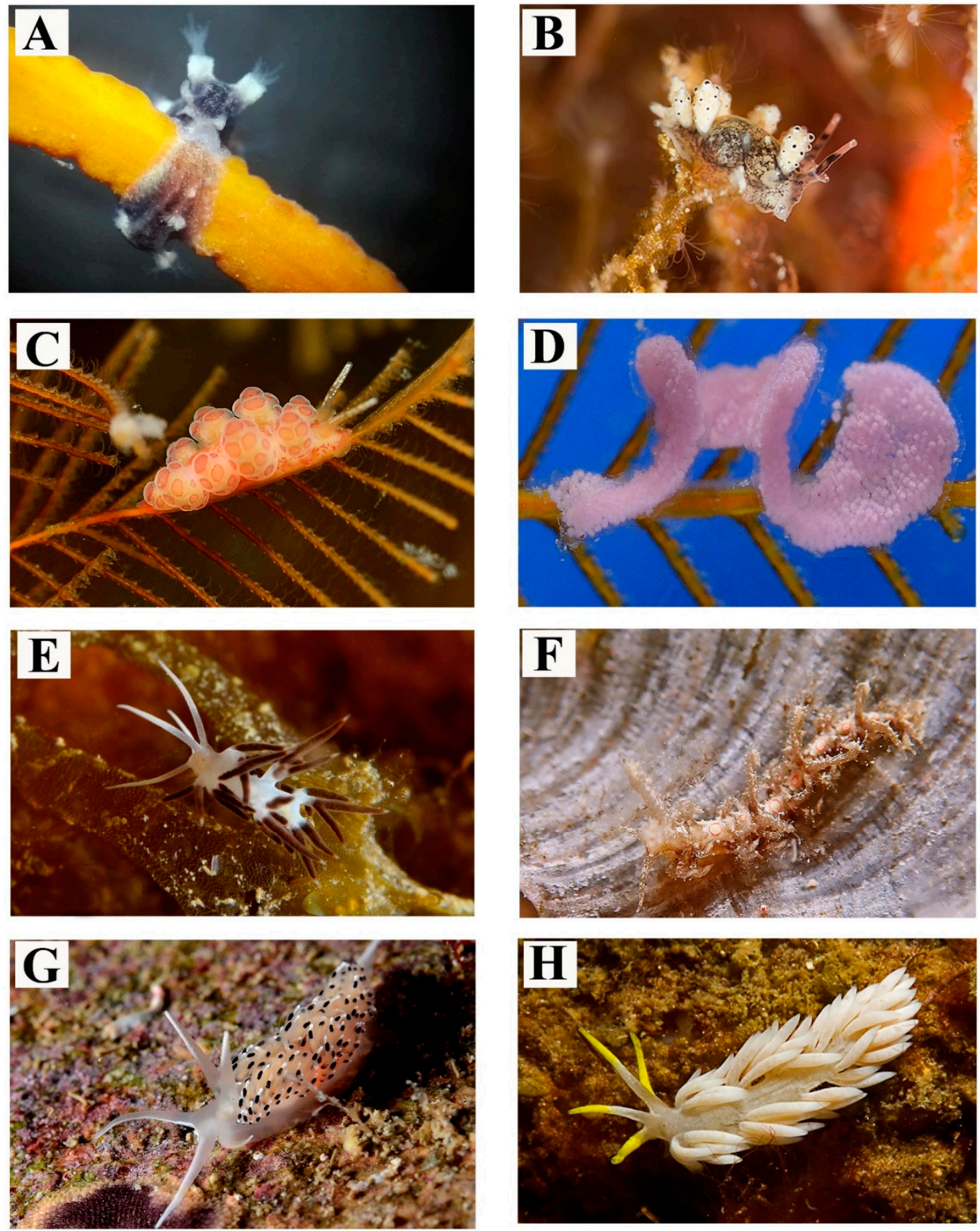

Figure 4. (A) Tritonia nilsodhneri. St. 18 (B) Doto cervicenigra. St. 11 (C) Doto floridicola. St. 22 (D) Spawn of Doto floridicola. St. 22 (E) Paraflabellina gabinierei. St. 11 (F) Limenandra nodosa. St. 3 (G) Caloria elegans. St. 11 (H) Dicata odhneri St. 11. 

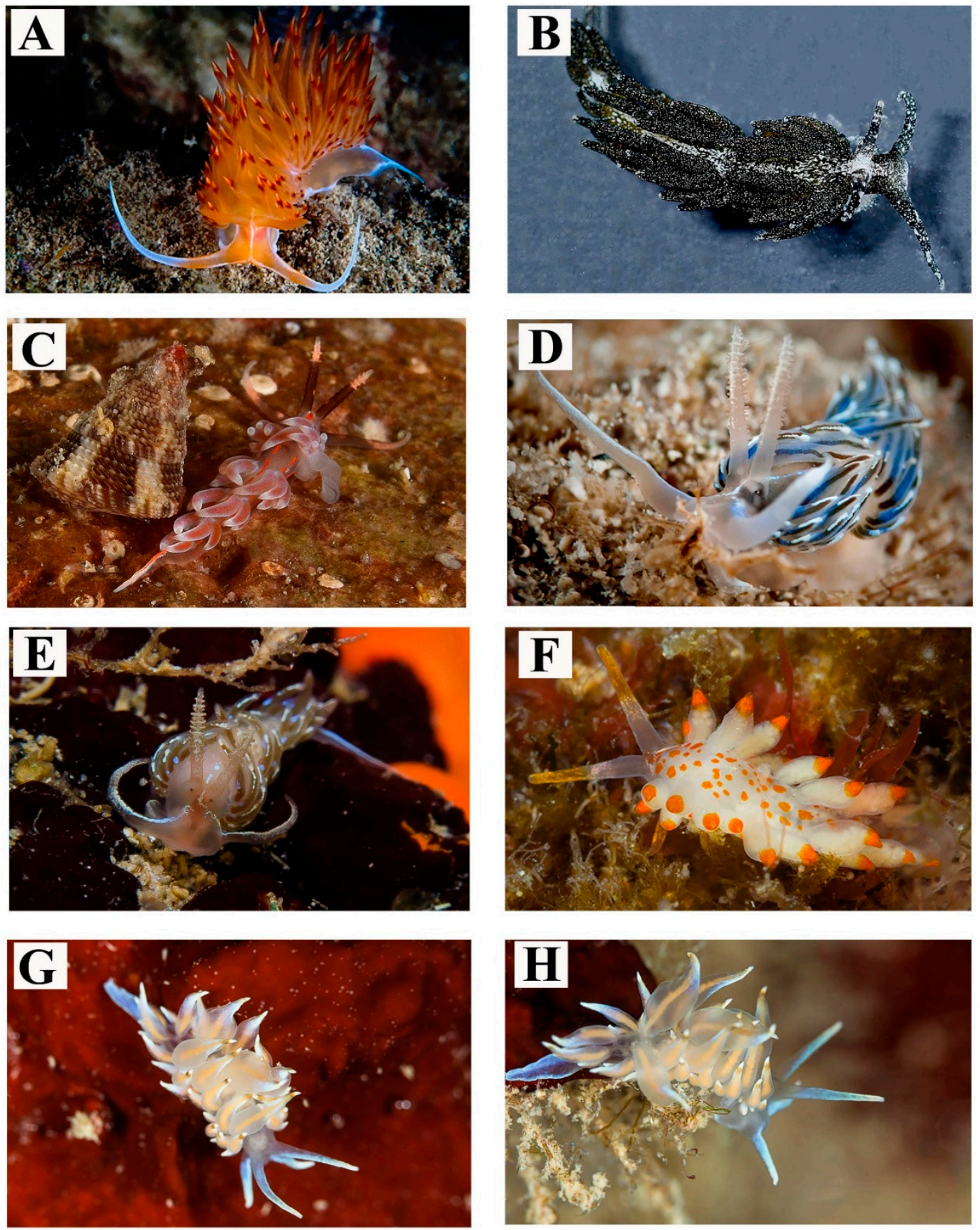

Figure 5. (A) Dondice banyulensis. St. 13 (B) Facelina fusca. St. 7 (C) Facelina rubrovittata. St. 11 (D,E) Facelina vicina. St. 11 (F) Eubranchus cf. farrani. St. 11 (G,H) Eubranchus cf. linensis St. 11. 

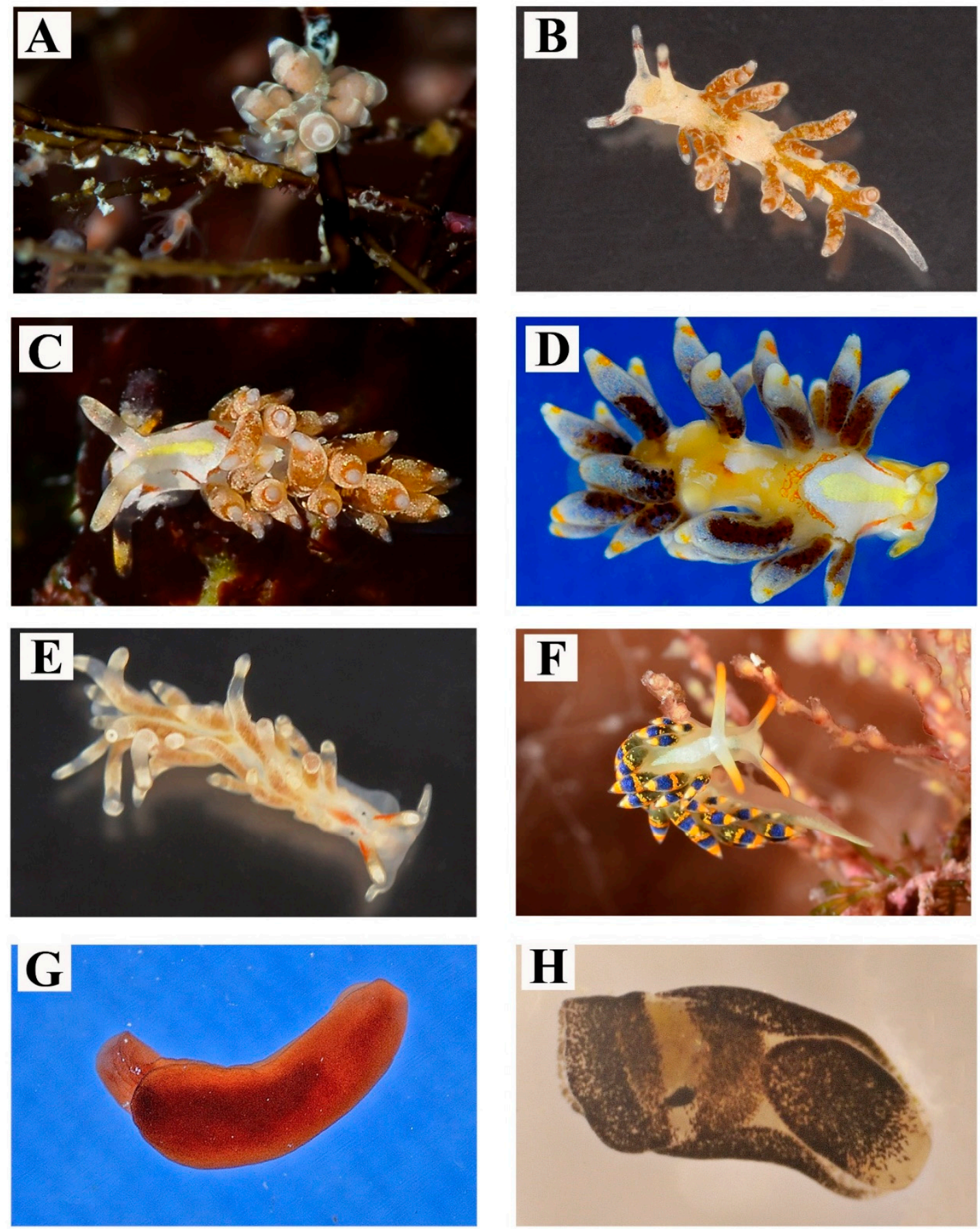

Figure 6. (A) Catriona maua. St. 9 (B) Rubramoena amoena. St. 22 (C) Trinchesia genovae. St. 11 (D) Trinchesia genovae. St. 7 (E) Trinchesia cf. miniostriata St. 22 (F) Trinchesia morrowae. St. 11 (G) Runcina cf. ferruginea. St. 22 (H) Philine punctata St. 9. 

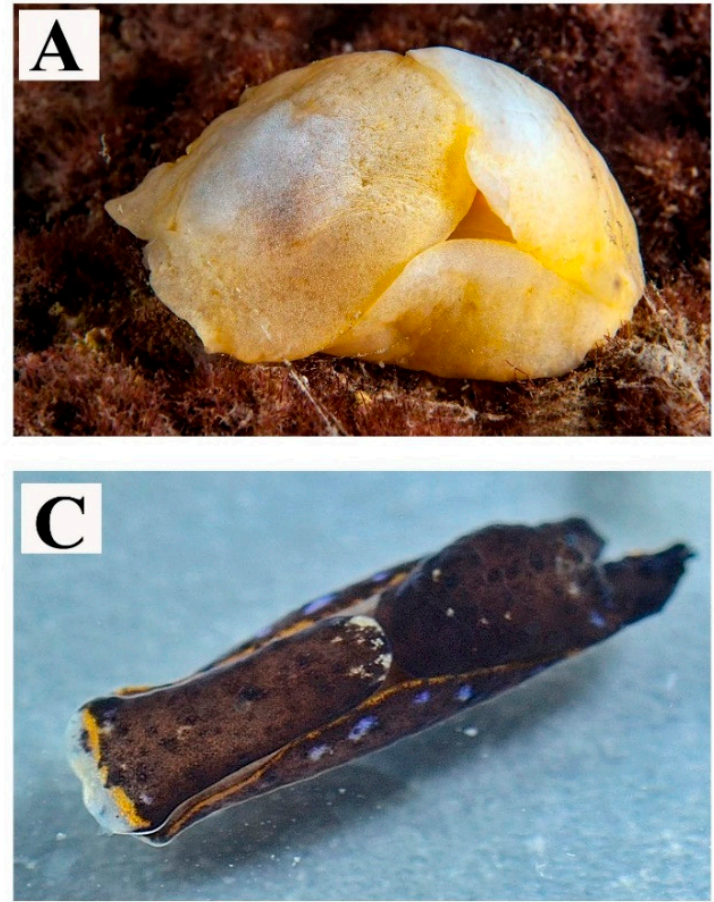

\section{E}
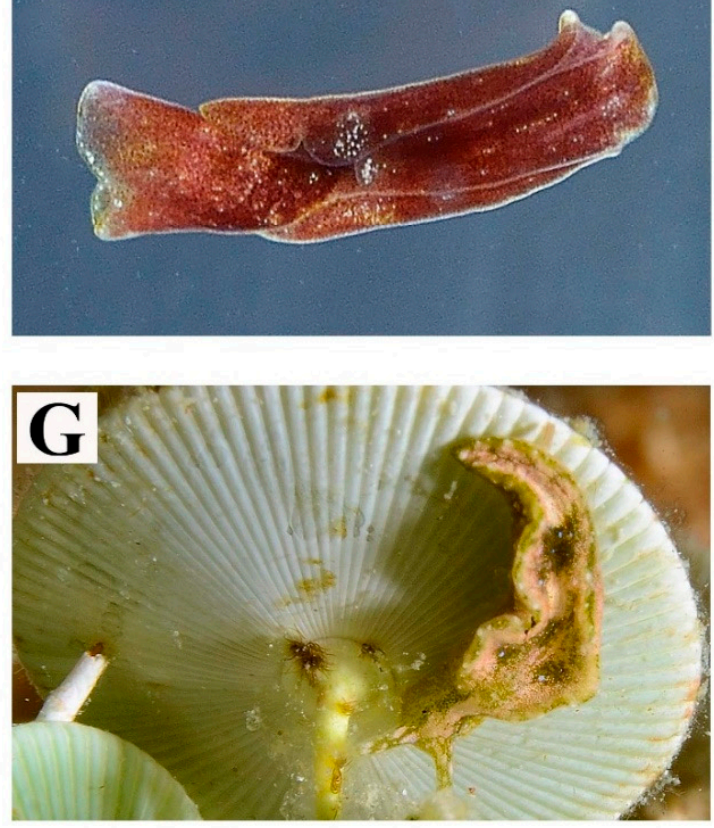

Figure 7. (A) Philine quadripartita. St. 1 (B) Camachoaglaja africana. St. 7 (C) Camachoaglaja africana. St. 22 (D) Philinopsis depicta. St. 10 (E) Melanochlamys wildpretii. St. 7 (F) Haminoea cf. orteai. St. 22 (G) Elysia gordanae. St. 11 (H) Elysia gordanae. St. 11.
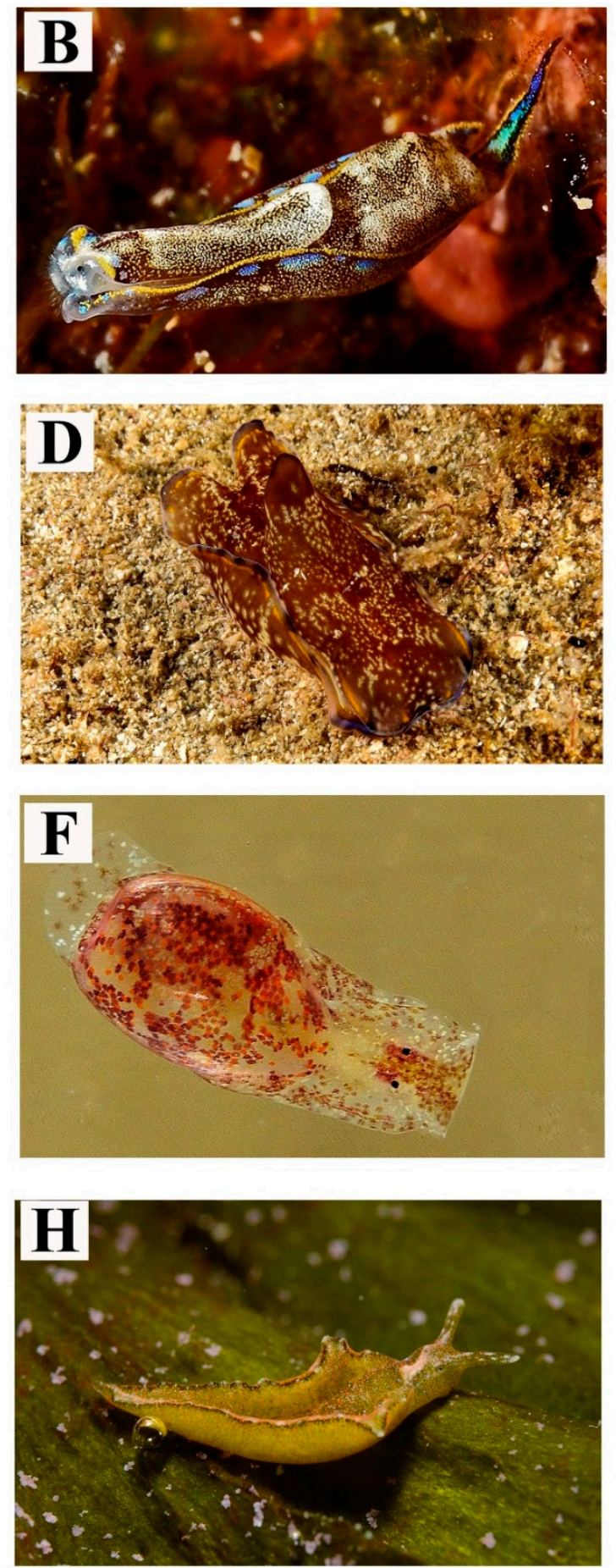

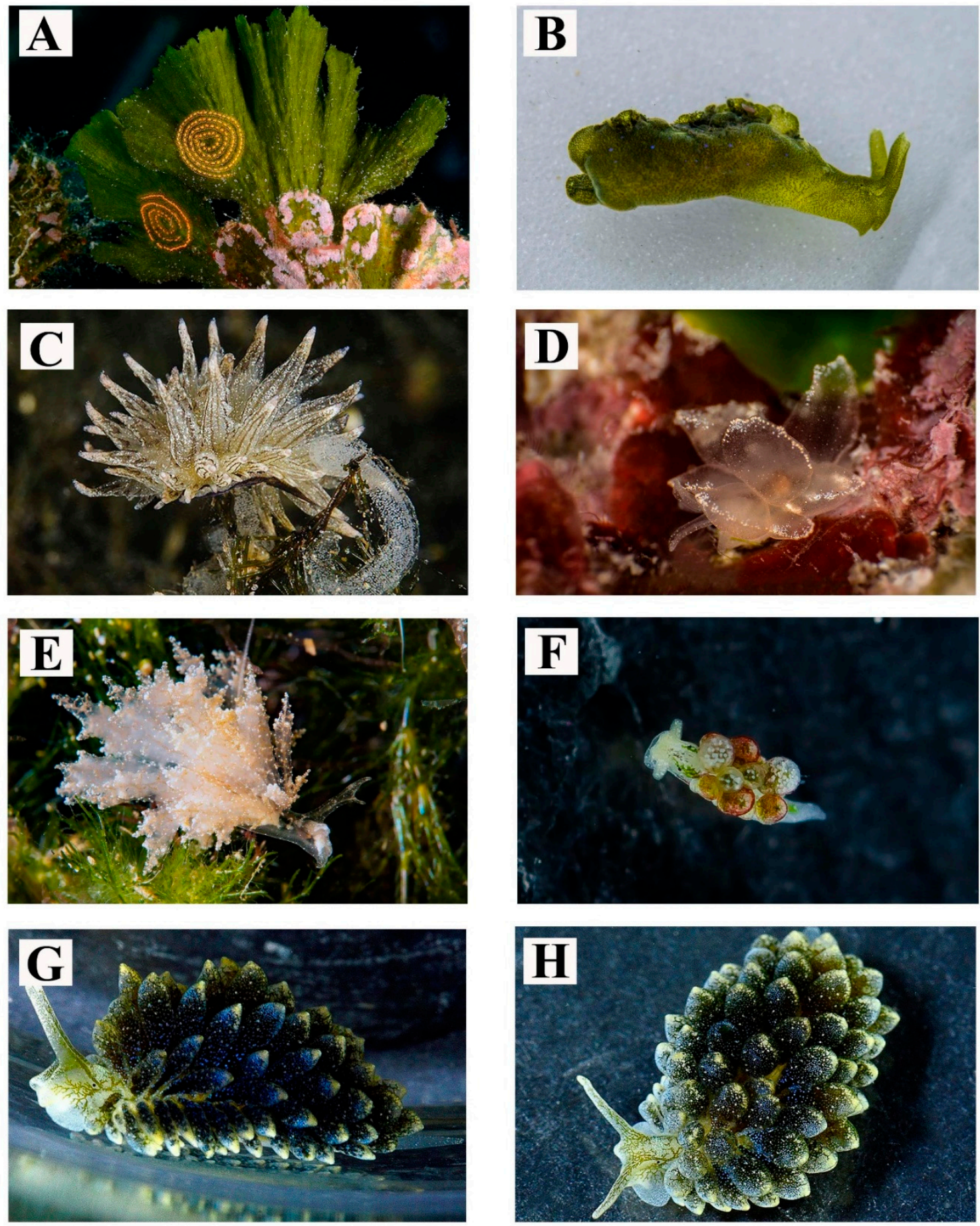

Figure 8. (A) Spawn of Elysia gordanae. St. 22 (B) Elysia margaritae. St. 11 (C) Aplysiopsis elegans. St. 1 (D) Cyerce graeca. St. 11 (E) Hermaea bifida. St. 1 (F) Hermaea paucicirra. St. 7 (G,H) Ercolania coerulea. St. 7 . 

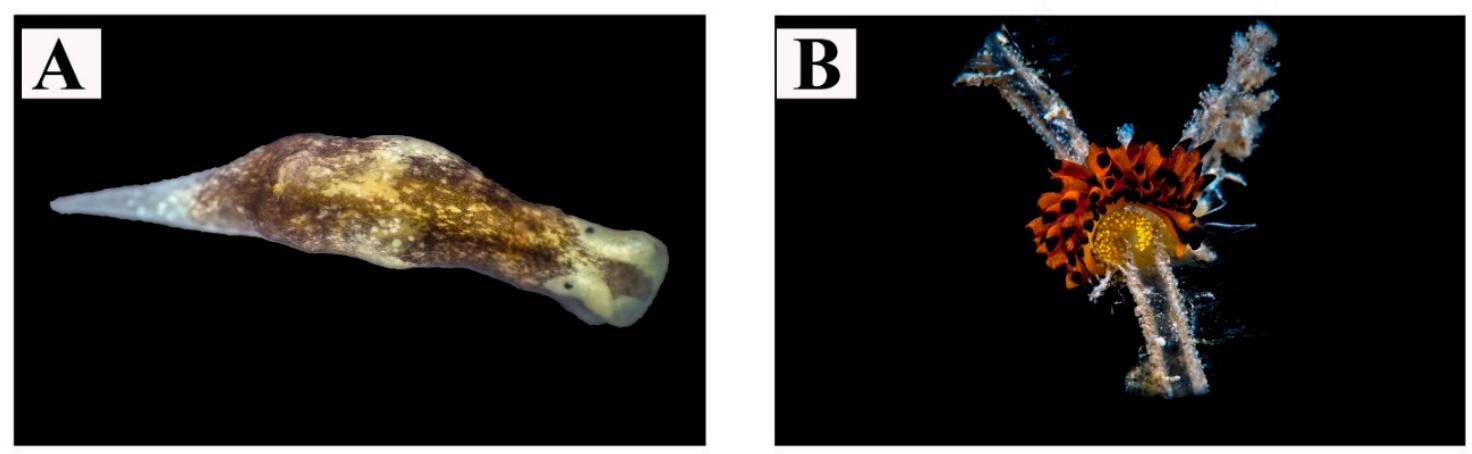

Figure 9. (A) Limapontia capitata. St. 24 (B) Placida cremoniana. St. 1.

\section{Discussion}

This study reported 160 marine Heterobranchia species from the Salento Peninsula giving the most up to date list of marine heterobranchs inhabiting this area of the Apulian coasts. This was particularly remarkable considering that the total number of registered species belonging to this mollusks group for the whole Mediterranean Sea was approximately 550 [51]. The composition of the main groups of marine heterobranchs living in the Salento Peninsula reported in this work is shown in Figure 10. The groups with the largest numbers of species added were Cladobranchia (18) and Doridina (9), as expected, these nudibranchs belonged to the groups with the richest variety of taxa. Surprisingly, with the present contribution, 6 species of Cephalaspidea were added to the ones reported previously. Another interesting consideration was concerning the superorder Sacoglossa, which almost doubled after this study. Finally, some species were noteworthy because they are currently under studied or because the validity of the species is still in doubt. This was the case for Berthellina cf. edwardsii, Eubranchus cf. exiguus, E. cf. farrani, E. cf. linensis, Facelina fusca, Haminoea cf. orteai, Runcina cf. ferruginea and Trinchesia cf. miniostriata. In particular, in the case of Berthellina cf. edwardsii we depicted (Figure 2B) the internal shell ( $4.2 \mathrm{~mm}$ long), since it fits the standard average length of the shell commonly used as diagnostic for this species, albeit we preferred to keep an uncertainty (cf.) before a further molecular study would allow a clear-cut identification. The studied marine area, deeply influenced by two different seas and characterized by a variety of submarine habitats, hosts a high variety of species, which makes it an important geographical area for this sea slug diversity. In fact, with this work we report that the total number of marine Heterobranchia living in the Salento Peninsula is 160, which is about a third of the total number of currently accepted species reported for the whole Mediterranean Sea. This is quite an important finding, also considering the fact that a high diversity in heterobranchs composition indirectly reflects a high structuring and diversification of the habitats involved, and consequently, of the biodiversity that they contribute to maintain [14]. Studying and monitoring the marine Heterobranchia diversity in the Salento Peninsula is valuable for highlighting the consequences of the global marine changes reported in the last decade [10-12,59,60], such as warming and acidification of waters or invasion of alien species. An additional detailed and constantly updated iconography is available on the website of the Salento Sommerso group (http://www.salentosommerso.it/index_opi.php), a non-profit association devoted to the preservation and documentation of the underwater biodiversity of the Salento Peninsula. 


\section{Composition of the main Heterobranchia groups}

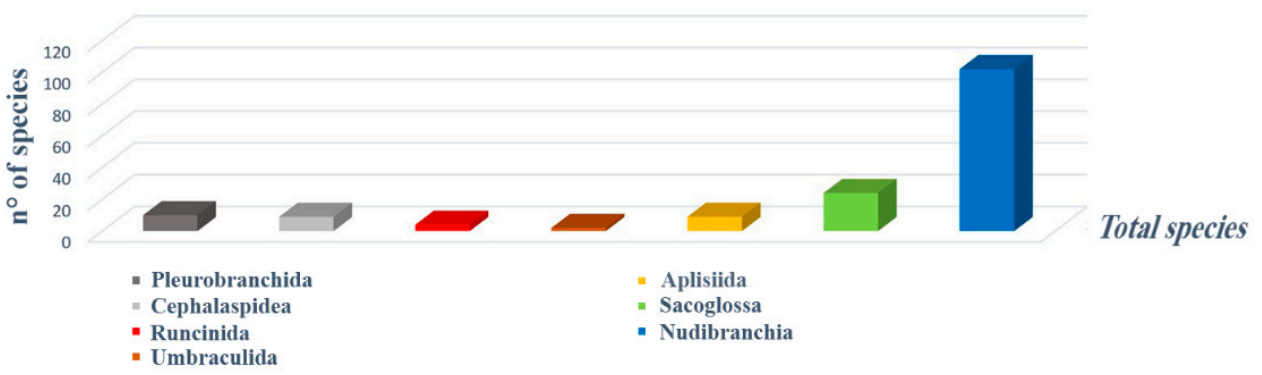

Figure 10. Comparison between the main heterobranch groups; the graph shows the comparison between the total species of the Salento Peninsula, clustered into the main groups.

\section{Conclusions}

The study of marine diversity is a fundamental topic especially if focused on areas like Mediterranean Sea, which is characterised by a high rate of endemic and cryptic species. In this study we have investigated the presence of marine Heterobranchia in the Salento Peninsula (Apulia, South-Italy) considering for the first time both Ionian and Adriatic coasts. Results of a nine-year study reports 160 species inhabiting the studied area with new records from the sublittoral waters, ecological notes, local distribution and systematic remarks. This inventory of marine Heterobranchia encountered in the Salento Peninsula adds 45 species to the previously known for this area. For all the added species, we have figured live animals mostly by in situ photographs to document species identification and reported data on ecology, phenotypical variability and abundance. This inventory of marine Heterobranchia provides a baseline for future monitoring of both coastal sides and could serve as a starting point for further molecular studies aiming to unveil Mediterranean cryptic diversity.

Author Contributions: F.V. and C.L. conceived the project and performed most of the SCUBA diving and took in situ pictures. G.F. and P.M. analysed the data and carried out the SCUBA diving. F.V., C.L., G.F., and P.M. wrote the manuscript. All authors have read and agreed to the published version of the manuscript.

Funding: Authors sincerely acknowledge the University of Roma Tre for financial support (CAL/2018 and CAL/2019).

Acknowledgments: Special thanks to Genuario Belmonte (University of Salento, Italy), Lucas Cervera Currado (University of Cadiz, Spain), and Egidio Trainito (Sardinia, Italy) for their useful suggestions. The authors wish to thank Elena Mazzone (Rome, Italy) for the revision of the English text. We would also like to thank Paolo D'Ambrosio, Director of the Porto Cesareo AMP, for permission to carry out research in the Marine Protected Area of Porto Cesareo (Lecce). The authors are greatly indebted to Marco Oliverio (Rome, Italy) for the revision of the English text. A very special thanks to our diving companions Marcella D'Elia, Domenico Licchelli, Cesare Bortone, and Pierantonio Cicirillo, with whom we shared many dives. We thank Antonello Perrone for suggestions and criticism. We thank Gianfranco Alemanno, Andrea Astore, Gianni Colucci, Piero Lenoci, Vincenzo Marra, Enrico Pati, and Gianluca Romano, for supporting us with many photographic images, specimens, and for sharing some of their data with us. Thanks to Manuel Ballesteros from the University of Barcelona (Spain), Marta Pola from the University of Madrid (Spain), Enric Madrenas and Miquel Pontes (Spain), Manuel Malaquias of the University of Bergen (Norway), and Jakov Prkić (Croatia) for the many suggestions and constructive exchanges of ideas and experiences. The authors are greatly indebted to the four anonymous reviewers, who provided many valuable comments, and improved the English.

Conflicts of Interest: The authors declare no conflict of interest. The funders had no role in the design of the study; in the collection, analyses, or interpretation of data; in the writing of the manuscript, or in the decision to publish the results.

\section{References}

1. Dainelli, G. Appunti Geologici sulla Parte Meridionale del Capo di Leuca; Tipografia della Pace di Filippo Cuggiani: Rome, Italy, 1901.

2. De Giorgi, C. La Serie Geologica dei Terreni nella Penisola Salentina; Tipografia della Pace di Filippo Cuggiani: Rome, Italy, 1903. 
3. Biasutti, R. Note morfologiche ed idrografiche sulla Terra d'Otranto. Riv. Geogr. It 1911, 18, 508-531.

4. Sacco, F. La geotettonica dell'Appennino meridionale. Boll. Soc. Geol. Ital. 1912, 31, 379-387.

5. Colamonico, C. La Geografia della Puglia: Profilo Monografico Regionale:(Con una Carta Geografica a Colori); Cressati: Bari, Italy, 1926.

6. $\quad$ Spalding, M.D.; Fox, H.E.; Allen, G.R.; Davidson, N.; Ferdaña, Z.A.; Finlayson, M.; Halpern, B.S.; Jorge, M.A.; Lombana, A.; Lourie, S.A.; et al. Notes Marine Ecoregions of the World: A Bioregionalization of Coastal and Shelf Areas. BioScience 2007, 57, 573-583. [CrossRef]

7. Bianchi, C.N. Proposta di suddivisione dei mari italiani in Settori Biogeografici. Not. Soc. Biol. Mar. 2004, 46, 57-59.

8. Taviani, M.; Angeletti, L.; Beuck, L.; Campiani, E.; Canese, S.; Foglini, F.; Freiwald, A.; Montagna, P.; Trincardi, F. Reprint of 'On and off the beaten track: Megafaunal sessile life and Adriatic cascading processes'. Mar. Geol. 2016, 375, 146-160. [CrossRef]

9. Savini, A.; Corselli, C. High-resolution bathymetry and acoustic geophysical data from Santa Maria di Leuca Cold Water Coral province (Northern Ionian Sea-Apulian continental slope). Deep Sea Res. Part II Trop. Stud. Oceanogr. 2010, 57, 326-344. [CrossRef]

10. Goddard, J.H.R.; Gosliner, T.M.; Pearse, J.S. Impacts associated with the recent range shift of the aeolid nudibranch Phidiana hiltoni (Mollusca, Opisthobranchia) in California. Mar. Biol. 2011, 158, 1095-1109. [CrossRef]

11. Goddard, J.H.R.; Treneman, N.; Pence, W.E.; Mason, D.E.; Dobry, P.M.; Green, B.; Hoover, C. Nudibranch Range Shifts associated with the 2014 Warm Anomaly in the Northeast Pacific. Bull. South. Calif. Acad. Sci. 2016, 115, 15-40. [CrossRef]

12. Goddard, J.H.R.; Treneman, N.; Prestholdt, T.; Hoover, C.; Green, B.; Pence, W.E.; Douglas, E.; Mason, D.E.; Dobry, P.L.; Sones, J.L.; et al. Heterobranch Sea Slugs range shifts in the northeast Pacific Ocean associated with the 2015-16 El Niño. Proc. Calif. Acad. Sci. 2018, 4, 107-131.

13. Nimbs, M.J.; Larkin, M.; Davis, T.R.; Harasti, D.; Willan, R.C.; Smith, D.A. Southern range extensions for 661 twelve heterobranch sea slugs (Gastropoda: Heterobranchia) on the eastern coast of Australia. Mar. Biodiv. Rec. 2016, 9, 27. [CrossRef]

14. Eisenbarth, J.H.; Undap, N.; Papu, A.; Schillo, D.; Dialao, J.; Reumschüssel, S.; Kaligis, F.; Bara, R.; Schäberle, T.F.; König, M.G.; et al. Marine Heterobranchia (Gastropoda, Mollusca) in Bunaken National Park, North Sulawesi, Indonesia-A Follow-Up Diversity Study. Diversity 2018, 10, 127. [CrossRef]

15. Cimino, G.; Ghiselin, M.T. Chemical defense and the evolution of opisthobranch gastropods. Proc. Calif. Acad. Sci. 2009, 60, 175-422.

16. Carbone, M.; Gavagnin, M.; Haber, M.; Guo, Y.-W.; Fontana, A.; Manzo, E.; Genta-Jouve, G.; Tsoukatou, G.; Rudman, W.B.; Cimino, G.; et al. Packaging and Delivery of Chemical Weapons: A Defensive Trojan Horse Stratagem in Chromodorid Nudibranchs. PLoS ONE 2013, 8, e62075. [CrossRef] [PubMed]

17. Cheney, K.L.; White, A.; Mudianta, I.W.; Winters, A.E.; Quezada, M.; Capon, R.J.; Mollo, E.; Garson, M.J. Choose your weaponry: Selective storage of a single toxic compound, Latrunculin A, by closely related Nudibranch Molluscs. PLoS ONE 2016, 11, e0145134. [CrossRef]

18. Winters, A.E.; White, A.M.; Dewi, A.S.; Mudianta, W.I.; Wilson, N.G.; Forster, L.C.; Garson, M.J.; Cheney, K.L. Distribution of defensive metabolites in Nudibranch Molluscs. J. Chem. Ecol. 2018, 44, 384-396. [CrossRef]

19. Winters, A.E.; Wilson, N.G.; van den Berg, C.P.; How, M.J.; Endler, J.A.; Marshall, N.J.; White, A.M.; Garson, M.J.; Cheney, K.L. Toxicity and taste: Unequal chemical defences in a mimicry ring. Proc. R. Soc. B 2018, 285, 20180457. [CrossRef]

20. Borsa, P. Allozyme, mitochondrial-DNA, and morphometric variability indicate cryptic species of anchovy (Engraulis encrasicolus). Biol. J. Linnean Soc. 2002, 75, 261-269. [CrossRef]

21. Calvo, M.; Templado, J.; Oliverio, M.; Machordom, A. Hidden Mediterranean biodiversity: Molecular evidence for a cryptic species complex within the reef building vermetid gastropod Dendropoma petraeum (Mollusca: Caenogastropoda). Biol. J. Linnean Soc. 2009, 96, 898-912. [CrossRef]

22. Claremont, M.; Reid, D.G.; Williams, S.T. Evolution of corallivory in the gastropod genus Drupella. Coral Reefs 2011, 30, 977-990. [CrossRef]

23. Barco, A.; Houart, R.; Bonomolo, G.; Crocetta, F.; Oliverio, M. Molecular data reveal cryptic lineages within the northeastern Atlantic and Mediterranean small mussel drills of the Ocinebrina edwardsii complex (Mollusca: Gastropoda: Muricidae). Zool. J. Linnean Soc. 2013, 169, 389-407. [CrossRef] 
24. Carmona, L.; Gosliner, T.M.; Pola, M.; Cervera, J.L. A Molecular approach to the Phylogenetic status of the Aeolid genus Babakina Roller, 1973 (Nudibranchia). J. Molluscan Stud. 2011, 77, 417-422. [CrossRef]

25. Furfaro, G.; Mariottini, P.; Modica, M.V.; Trainito, E.; Doneddu, M.; Oliverio, M. Sympatric sibling species: The case of Caloria elegans and Facelina quatrefagesi (Gastropoda: Nudibranchia). Sci. Mar. 2016, 80, 511-520. [CrossRef]

26. Furfaro, G.; Modica, M.V.; Oliverio, M.; Mariottini, P. A DNA-barcoding approach to the phenotypic diversity of Mediterranean species of Felimare Ev. Marcus and Er. Marcus, 1967 (Mollusca: Gastropoda), with a preliminary phylogenetic analysis. Ital. J. Zool. 2016, 83, 195-207. [CrossRef]

27. Furfaro, G.; Salvi, D.; Mancini, E.; Mariottini, P. A multilocus view on Mediterranean aeolid nudibranchs (Mollusca): Systematics and cryptic diversity of Flabellinidae and Piseinotecidae. Mol. Phylogenet. Evol. 2018, 118, 13-22. [CrossRef] [PubMed]

28. Furfaro, G.; Mariottini, P. Less rare than we thought: Two new localities for Piseinotecus soussi Tamsouri, Carmona, Moukrim, Cervera, 2014 along the Tyrrhenian coast. Turk. J. Zool. 2019, 43, 287-289. [CrossRef]

29. Martín-Hervás, M.R.; Carmona, L.; Jensen, K.R.; Licchelli, C.; Vitale, F.; Cervera, J.L. Description of a new pseudocryptic species of Elysia Risso, 1818 (Heterobranchia, Sacoglossa) in the Mediterranean Sea. Bull. Mar. Sci. 2019, 96, 127-144. [CrossRef]

30. Perrone, A.S. Nudibranchi del genere Taringa Marcus, 1955 dal Golfo di Taranto (Heterobranchia: Nudibranchia). Boll. Malacol. 1992, 28, 207-220.

31. Perrone, A.S. Opistobranchi (Aplysiomorpha, Pleurobrancomorpha, Sacoglossa, Nudibranchia) del litorale salentino (Mar Jonio) (Elenco-contributo primo). Thalassia Salentina 1983, 13, 118-144.

32. Perrone, A.S. Opistobranchi (Aplysiomorpha, Pleurobrancomorpha, Sacoglossa, Nudibranchia) del litorale salentino (Mare Jonio) (Elenco-Contributo secondo). Thalassia Salentina 1986, 16, 19-42.

33. Perrone, A.S. Primo rinvenimento di Elysia flava Verrill, 1901 per le coste italiane (Heterobranchia: Sacoglossa). Atti Soc. Ital. Sci. Nat. 1988, 129, 483-488.

34. Perrone, A.S. Una nuova specie di Elysiidae, Elysia hetta nov. sp. dal litorale salentino (Mediterraneo-Golfo di Taranto) (Heterobranchia: Sacoglossa). Atti Soc. Ital. Sci. Nat. 1990, 130, 249-252.

35. Perrone, A.S. Una nuova specie di Nudibranchi dal Golfo di Taranto: Rostanga anthelia nov. sp. (Heterobranchia: Nudibranchia). Boll. Malacol. 1990, 26, 179-188.

36. Perrone, A.S. Una nuova specie di Nudibranchi Doridiani, Peltodoris sordii nov. sp., dalla biocenosi a Anadiomene stellata, Geodia cydonium e Holothuria impatiens. Boll. Malacol. 1989, 25, 295-300.

37. Perrone, A.S. Una specie di Nudibranchi nuova per le coste italiane: Ridescrizione di Geitodoris (Verrilia) bonosi Ortea \& Ballesteros, 1981 (Heterobranchia: Nudibranchia). Boll. Malacol. 1992, 28, 27-34.

38. Onorato, M.; Belmonte, G. Submarine caves of the Salento peninsula: Faunal aspects. Thalassia Salentina 2017, 39, 47-72.

39. Micaroni, V.; Strano, F.; Di Franco, D.; Crocetta, F.; Grech, D.; Piraino, S.; Boero, F. Project “Biodiversity MARE Tricase": A biodiversity inventory of the coastal area of Tricase (Ionian Sea, Italy)—Mollusca: Heterobranchia. Eur. Zool. J. 2018, 85, 180-193. [CrossRef]

40. Colucci, G.; Strafella, R.; Trainito, E.; Doneddu, M. First record of the genus Dermatobranchus van Hasselt, 1824, in the Mediterranean Sea (Nudibranchia: Arminidae). Mediterr. Mar. Sci. 2015, 16, 331-333. [CrossRef]

41. Pola, M.; Paz-Sedano, S.; Macali, A.; Minchin, D.; Marchini, A.; Vitale, F.; Licchelli, C.; Crocetta, F. What is really out there? Review of the genus Okenia Menke, 1830 (Nudibranchia: Goniodorididae) in the Mediterranean Sea with description of two new species. PLoS ONE 2019, 14, e0215037. [CrossRef]

42. Araujo, A.K.; Pola, M.; Malaquias, M.A.E.; Cervera, J.L. To be or not to be? What molecules say about Runcina brenkoae Thompson, 1980 (Gastropoda: Heterobranchia: Runcinida). Sci. Mar. 2019, 83, 223-235. [CrossRef]

43. Golestani, H.; Crocetta, F.; Padula, V.; Camacho-García, Y.; Langeneck, J.; Poursanidis, D.; Pola, M.; Yokeş, M.B.; Cervera, J.L.; Jung, D.W.; et al. The little Aplysia coming of age: From one species to a complex of species complexes in Aplysia parvula (Mollusca: Gastropoda: Heterobranchia). Zool. J. Linnean Soc. 2019, 187, 279-330. [CrossRef]

44. Korshunova, T.; Malmberg, K.; Prkić, J.; Petani, A.; Fletcher, K.; Lundin, K.; Martynov, A. Fine-scale species delimitation: Speciation in process and periodic patterns in nudibranch diversity. ZooKeys 2020, 917, 15-50. [CrossRef] [PubMed]

45. Nimbs, M.J.; Smith, S.D. An illustrated inventory of the sea slugs of New South Wales, Australia (Gastropoda: Heterobranchia). Proc. R. Soc. Vic. 2016, 128, 44-113. [CrossRef] 
46. Smith, S.D.; Davis, T.R. Slugging it out for science: Volunteers provide valuable data on the diversity and distribution of heterobranch sea slugs. Molluscan Res. 2019, 39, 214-223. [CrossRef]

47. Smith, S.D.; Nimbs, M.J. Quantifying temporal variation in heterobranch (Mollusca: Gastropoda) sea slug assemblages: Tests of alternate models. Molluscan Res. 2017, 37, 140-147. [CrossRef]

48. iNaturalist. Available online: https://www.inaturalist.org/ (accessed on 19 April 2020).

49. Word Register of Marine Species. Available online: http://www.marinespecies.org/ (accessed on 21 April 2020).

50. Cattaneo-Vietti, R.; Chemello, R.; Giannuzzi-Savelli, R. Atlante dei Nudibranchi del Mediterraneo; Editrice La Conchiglia: Rome, Italy, 1990.

51. Trainito, E.; Doneddu, M. Nudibranchi del Mediterraneo; Il Castello: Milan, Italy, 2014.

52. OPK Opistobranquis. Available online: https://opistobranquis.info/en/ (accessed on 19 April 2020).

53. Sea Slug Forum. Available online: http://www.seaslugforum.net/ (accessed on 19 April 2020).

54. Furfaro, G.; Picton, B.; Martynov, A.; Mariottini, P. Diaphorodoris alba Portmann \& Sandmeier, 1960 is a valid species: Molecular and morphological comparison with D. luteocincta (M. Sars, 1870) (Gastropoda: Nudibranchia). Zootaxa 2016, 4193, 304-316.

55. Mastrototaro, F.; Panetta, P.; D'Onghia, G. Further records of Melibe viridis (Mollusca, Nudibranchia) in the Mediterranean Sea, with observations on the spawning. Vie Milieu 2004, 54, 251-253.

56. Ballesteros, M.; Madrenas, E.; Pontes, M. “Doto Fragaria” in OPK-Opistobranquis (2012-2020). Available online: https://opistobranquis.info/en/hejyE (accessed on 21 April 2020).

57. Tamsouri, N.; Carmona, L.; Moukrim, A.; Cervera, J.L. Description of a new species of Piseinotecus (Castropoda, Heterobranchia, Piseinotecidae) from the northeastern Atlantic Ocean. Bull. Mar. Sci. 2014, 90, 991-997. [CrossRef]

58. Crocetta, F.; Colamonaco, G. Percnon gibbesi (Crustacea: Decapoda) and Aplysia dactylomela (Mollusca: Gastropoda) in the Taranto Gulf (Italy, Ionian Sea): New populations incoming. Mar. Biodiv. Rec. 2010, 3, e88. [CrossRef]

59. Wernberg, T.; Russell, B.D.; Moore, P.J.; Ling, S.D.; Smale, D.A.; Campbell, A.; Coleman, M.A.; Steinberg, P.D.; Kendrick, G.A.; Connell, S.D. Impacts of climate change in a global hotspot for temperate marine biodiversity and ocean warming. J. Exp. Mar. Biol. Ecol. 2011, 400,7-16. [CrossRef]

60. Wernberg, T.; Smale, D.A.; Thomsen, M.S. A decade of climate change experiments on marine organisms: Procedures, patterns and problems. Glob. Change Biol. 2012, 18, 1491-1498. [CrossRef] 